\title{
Reductive Stress Causes Pathological Cardiac Remodeling and Diastolic Dysfunction
}

\author{
Gobinath Shanmugam, ${ }^{1, *}$ Ding Wang, ${ }^{2,{ }^{*}}$ Sellamuthu S. Gounder, ${ }^{3}$ Jolyn Fernandes, ${ }^{4}$ \\ Silvio H. Litovsky, ${ }^{1}$ Kevin Whitehead, ${ }^{3}$ Rajesh Kumar Radhakrishnan, ${ }^{1}$ Sarah Franklin, ${ }^{3}$ John R. Hoidal, ${ }^{5}$ \\ Thomas W. Kensler, ${ }^{6}$ Louis Dell'Italia, ${ }^{7}$ Victor Darley-Usmar, ${ }^{8}$ E. Dale Abel, ${ }^{9}$ Dean P. Jones, ${ }^{4}$ \\ Peipei Ping, ${ }^{2,10}$ and Namakkal S. Rajasekaran ${ }^{1,3,8,11, *}$
}

Aims: Redox homeostasis is tightly controlled and regulates key cellular signaling pathways. The cell's antioxidant response provides a natural defense against oxidative stress, but excessive antioxidant generation leads to reductive stress (RS). This study elucidated how chronic RS, caused by constitutive activation of nuclear erythroid related factor-2 (caNrf2)-dependent antioxidant system, drives pathological myocardial remodeling. Results: Upregulation of antioxidant transcripts and proteins in caNrf2-TG hearts (TGL and TGH; transgeniclow and -high) dose dependently increased glutathione (GSH) redox potential and resulted in RS, which over time caused pathological cardiac remodeling identified as hypertrophic cardiomyopathy (HCM) with abnormally increased ejection fraction and diastolic dysfunction in TGH mice at 6 months of age. While the TGH mice exhibited $60 \%$ mortality at 18 months of age, the rate of survival in TGL was comparable with nontransgenic (NTG) littermates. Moreover, TGH mice had severe cardiac remodeling at $\sim 6$ months of age, while TGL mice did not develop comparable phenotypes until 15 months, suggesting that even moderate RS may lead to irreversible damages of the heart over time. Pharmacologically blocking GSH biosynthesis using BSO (L-buthionine-SR-sulfoximine) at an early age ( $\sim 1.5$ months) prevented RS and rescued the TGH mice from pathological cardiac remodeling. Here we demonstrate that chronic RS causes pathological cardiomyopathy with diastolic dysfunction in mice due to sustained activation of antioxidant signaling.

Innovation and Conclusion: Our findings demonstrate that chronic RS is intolerable and adequate to induce heart failure (HF). Antioxidant-based therapeutic approaches for human HF should consider a thorough evaluation of redox state before the treatment. Antioxid. Redox Signal. 32, 1293-1312.

Keywords: reductive stress, Nrf2, cardiac hypertrophy, diastolic dysfunction, HCMiEF

\footnotetext{
${ }^{1}$ Cardiac Aging and Redox Signaling Laboratory, Division of Molecular and Cellular Pathology, Department of Pathology, University of Alabama at Birmingham, Birmingham, Alabama, USA.

${ }^{2}$ Department of Physiology, NIH BD2K Center of Excellence for Biomedical Computing at UCLA, University of California, Los Angeles, California, USA.

${ }^{3}$ Division of Cardiovascular Medicine and ${ }^{5}$ Pulmonary Medicine, University of Utah School of Medicine, Salt Lake City, Utah, USA.

${ }^{4}$ Division of Pulmonary, Allergy, Critical Care and Sleep Medicine, Emory University, Atlanta, Georgia, USA.

${ }^{6}$ Fred Hutch Cancer Research Center, Seattle, Washington, USA.

${ }^{7}$ Comprehensive Cardiovascular Center, School of Medicine, University of Alabama at Birmingham, Birmingham, Alabama, USA.

${ }^{8}$ Center for Free Radical Biology, University of Alabama at Birmingham, Birmingham, Alabama, USA.

${ }^{9}$ Division of Endocrinology and Metabolism, Fraternal Order of Eagles Diabetes Research Center, Roy J. and Lucille A. Carver College of Medicine, University of Iowa, Iowa City, Iowa, USA.

${ }^{10}$ Department of Medicine/Cardiology, NHLBI Integrated Cardiovascular Data Science Training Program at UCLA, Bioinformatics and Medical Informatics, and Scalable Analytics Institute (ScAi) at UCLA School of Engineering, Los Angeles, California, USA.

${ }^{11}$ Department of Biomedical Engineering, University of Alabama at Birmingham, Birmingham, Alabama, USA.

*These authors contributed equally to this work.
} 


\section{Innovation}

Constitutive activation of nuclear erythroid related factor- 2 in the hearts of transgenic mice increased the endogenous antioxidants and caused reductive stress (RS). Specifically, the RS mice (transgenic-high) developed hypertrophic cardiomyopathy with abnormally increased ejection fraction. The transition from compensated hypertrophy to diastolic dysfunction and heart failure, as evidenced in transgenic-low mice with increased age $(>15$ months), signifies a pathological outcome over time. The characterization of biochemical and functional responses to $\mathrm{RS}$ in the heart indicates the potential for damage due to excessive antioxidant signaling. Pharmacologic depletion of glutathione prevents RS-induced cardiac remodeling and dysfunction.

\section{Introduction}

$\mathbf{H}$ EART FAILURE (HF) is a frequent cause of human morbidity and mortality worldwide $(10,14)$. Given the heart's limited regenerative capacity, restoring cardiac function following maladaptive remodeling is a major challenge (61). Although inherited mutations account for most hypertrophic cardiomyopathy (HCM) events $(37,48)$, the molecular mechanisms underlying noninherited HCMs are poorly understood. Many heart diseases are linked to oxidative stress (20,
49, 63, 64); augmenting antioxidants and modulating redoxsensitive targets have been thought to protect the heart against oxidative stress $(16,50,51,55)$. Since free radical scavenging antioxidants have largely failed to provide cardioprotective benefit $(2,9,15,30,59)$, activation of endogenous antioxidant systems has been advocated as a possible alternative $(1,12,34$, 57). However, the threshold for antioxidant activation to deliver beneficial effects, and its long-term impact on the heart, is largely unknown. Therefore, investigating the biochemical and physiological responses of the heart under moderate or extreme reductive redox conditions may offer opportunities to identify novel mechanisms with clinical significance.

Nuclear factor (erythroid-derived-2)-like 2 (NFE2L2 or Nrf2) is a stress-inducible transcription factor that binds to the antioxidant response element (ARE) and regulates its target expression $(38,40,41,62)$. Under basal conditions, $\mathrm{Nrf} 2$ is retained in the cytoplasm by its repressor Keap 1 and post-translationally marked for degradation $(17,27,29)$. Under stress conditions, the oxidative-sensitive modifications on Keap1 cysteine residues impede the polyubiquitination of $\mathrm{Nrf} 2$, allowing the nuclear translocation of Nrf2 to transactivate antioxidant genes $(8,27,31,40$, 58). Moreover, de novo activation of the Nrf2 gene by mutations was found in patients with multisystem disorders, leading to a sustained upregulation of the Nrf2dependent antioxidant system (21). Therefore, here we investigated whether a sustained activation of antioxidant systems leads to RS, and how chronic RS affects cardiac structure and function.
A

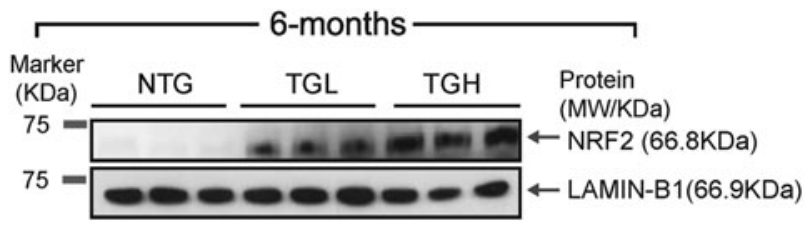

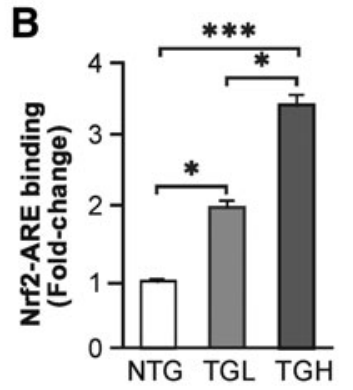

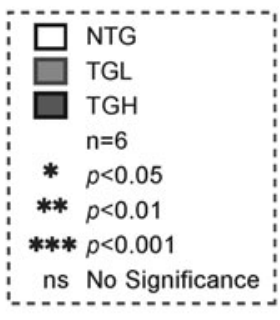

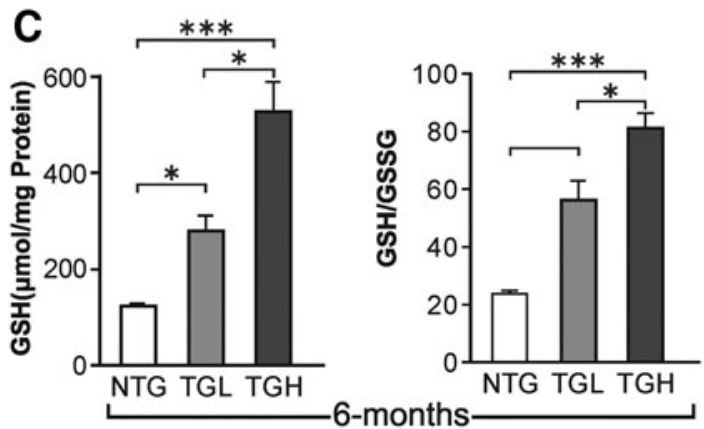
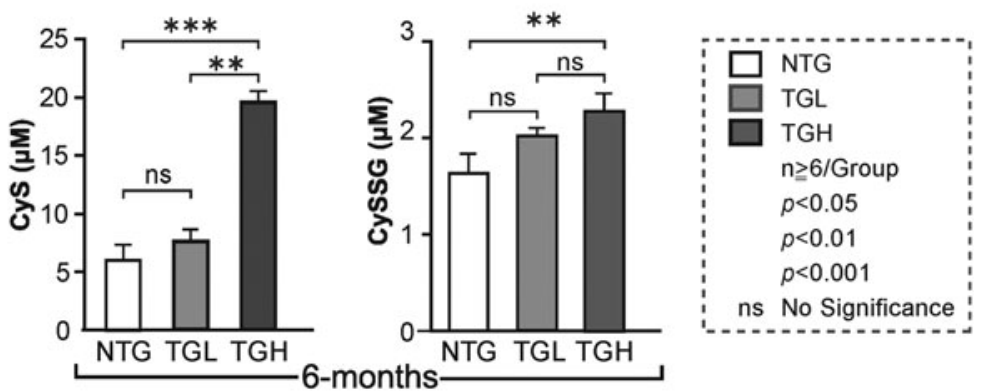

FIG. 1. Constitutive activation of Nrf2 induces chronic reductive stress in TG mouse hearts. (A) The nuclear Nrf2 levels in the heart were determined by IB in NTG and caNrf2 TGL and TGH mice $(n=3)$. Raw images (uncut) are represented in Supplementary Fig. S5. (B) The Nrf2 and Nrf2-ARE binding activities were measured in three mouse groups at 6-8 months of age $(n=6)$ using nuclear extracts. Results are presented as fold-change compared with the NTG group. (C) Myocardial levels of glutathione $(\mathrm{GSH})$ and the ratio of its reduced/oxidized form (GSH/GSSG) were determined by enzyme kinetic assays. In parallel, tissue concentrations of cysteine (Cys) and cysteine-GSH adduct (CySSG) were quantified by an HPLC-based fluorometric assay among the three mouse groups at 6-8 months of age ( $n=5 /$ group). Data are represented as mean \pm SEM. Significance: ${ }^{*} p<0.05 ; * * p<0.01$; *** $p<0.001$; ns, no significance. ARE, antioxidant response element; caNrf2, constitutive activation of nuclear erythroid-related factor-2; HPLC, high-performance liquid chromatography; IB, immunoblotting; NTG, nontransgenic; SEM, standard error of the mean; TG, transgenic; TGH, transgenic high; TGL, transgenic high. 

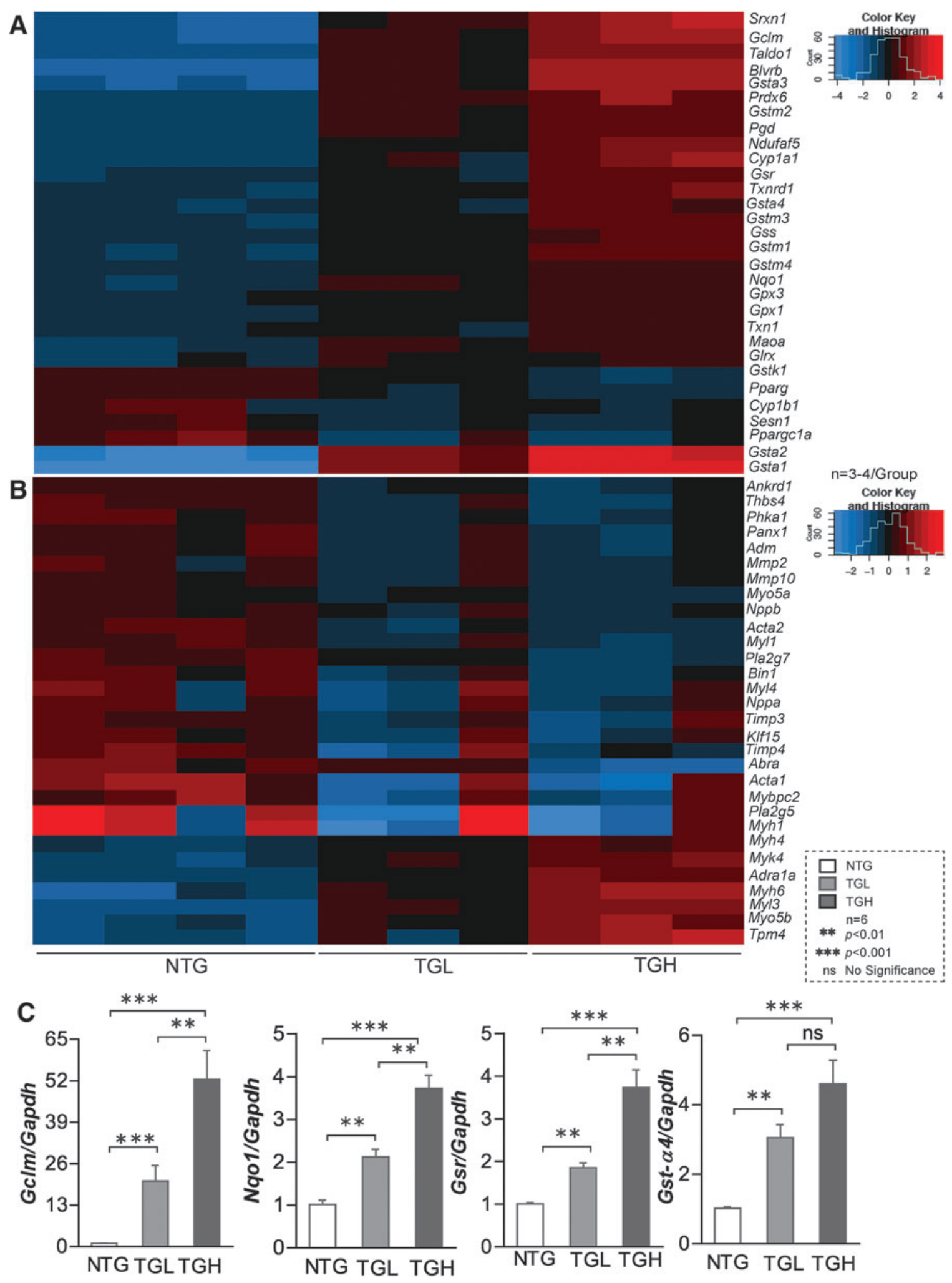

FIG. 2. caNrf2 expression induces transcriptional changes in mouse myocardium. Heat map illustrating RNA sequencing data of RS myocardium describing $\log _{2}$ expression changes in genes involved in (A) glutathione metabolism and (B) myocardial development in NTG and TG mice at $6-8$ months of age $(n=3-4$ /group). These data clusters are generated using original transcriptome data from NTG and TG (Low \& High) mice. (C) Transcripts involved in glutathione metabolism were randomly selected and validated using qPCR ( $n=5$ mice/group). Data are represented as mean \pm SEM. Significance: $* * p<0.01$; $* * * p<0.001$; ns, no significance. qPCR, quantitative polymerase chain reaction; RS, reductive stress. Color images are available online. 
In this investigation, using a novel constitutive activation Nrf2 transgenic (caNrf2-TG) mouse model (53), we determined whether chronic RS is sufficient to cause myocardial hypertrophy and pathological cardiac remodeling. We discovered that sustained activity of Nrf2 in the heart perturbs cellular redox systems, resulting in chronic RS. Chronic RS induces functional changes at an early age that progress to irreversible pathological hypertrophy at later ages (6-8 months). This hypertrophy is initially accompanied by an abnormally increased ejection fraction (EF). Subsequently, chronic RS induces diastolic dysfunction and impaired ventricular relaxation leading to $\mathrm{HF}$ and reduced survival. In an effort to prevent RS, we used L-buthionine-SR-sulfoximine (BSO), an inhibitor of gamma-glutamylcysteine synthetase to pharmacologically block glutathione (GSH) biosynthesis (19, $22,44,46)$. Depletion of GSH in the caNrf2-TG mice diminished RS and delayed the onset of cardiac remodeling. Our findings have important implications for patients with $\mathrm{HCM}$ and progressive HF, particularly those receiving treatment aimed at activating endogenous antioxidant responses.

\section{Results}

\section{caNrf2 induces chronic $R S$ in mouse heart}

Clinical trials with agents intended to protect against oxidative stress in humans have often resulted in conflicting results $(9,30)$. The mechanistic underpinnings for these inconsistent effects in heart diseases are largely unknown, in part, due to lack of explicit and suitable in vivo models. To address this gap, we established heart-specific genetic mouse models that provide varied levels of caNrf2, a master regulator of antioxidants and the redox state (53). Using the mouse alpha-myosin heavy chain $(\alpha-\mathrm{MHC})$ promoter to direct the expression of caNrf2 in the heart, we recently established two different transgenic lines that express either low or high levels of caNrf2 at a young age ( $\sim 2$ months) (53). Cardiac-specific expression of $\mathrm{Nrf} 2$ protein was significantly increased in a transgene dose-dependent manner (Fig. 1A), along with augmented Nrf2-ARE (DNA) binding levels in both transgeniclow (TGL) and transgenic-high (TGH) mice (Fig. 1B). In light of the significant and transgene dose-dependent increase in Nrf2-ARE (DNA) binding in TG mice, we analyzed the myocardial redox state in the NTG and TG mice at 6 months of age $(23,25)$. The levels of the reduced form of GSH (Fig. 1C) and the myocardial GSH/GSSG ratio (Fig. 1C) were significantly increased in the TGH hearts at 6 months of age. Furthermore, the myocardial concentrations of cysteine, a rate- limiting precursor for GSH, were elevated by $150 \%$ in TGH hearts (Fig. 1C) as was its GSH-adduct (Cys-SGSH; Fig. 1C), while the levels were unchanged or not significantly different in TGL animals compared with NTG controls.

We then performed RNA sequencing in these experimental groups and results showed transgene dose-dependent expression of various antioxidant genes in TGL and TGH hearts, which were validated by quantitative polymerase chain reaction (qPCR) for randomly selected genes (Fig. 2A, C). At 6-8 months of age, Gclc (2.0-fold vs. 3.5-fold), Gclm (15-fold vs. 52-fold), Gsr (1.5-fold vs. 4-fold), Nqol (2-fold vs. 4-fold), Gst- $\alpha$ (3-fold vs. 4-fold), Gst- $\mu$ (3-fold vs. 4.2fold), Gpxl (1.0-fold vs. 1.5-fold), and catalase (1.0-fold $v s$. 1.5-fold) were significantly increased in TGL and TGH relative to NTG mouse hearts. These results indicate a dosedependent response for many antioxidant genes $(42,53)$.

Next, we determined whether the transcriptional changes induced by caNrf2 reflected on protein expression of these antioxidant enzymes. Immunoblot analysis of myocardial cytosol revealed a marked increase in the expression of various antioxidant proteins (Fig. 3A-F). At 6 months of age, glutamate-cysteine ligase catalytic (GCLC) (1.5-fold vs. 2.0fold), glutamate-cysteine ligase modifier (GCLM) (4-fold vs. 7-fold), glutathione reductase (GSR) (5-fold vs. 10-fold), NAD(P)H:Quinone Oxidoreductase 1 (NQO1) (2-fold vs. 4-fold), glutathione- $S$-transferase $\mathrm{Mu}$ (class) (GST- $\mu$ ) (8fold vs. 9-fold), glutathione peroxidase 1 (GPX1) (2-fold vs. 1.5-fold), and catalase (CAT) (3-fold vs. 5-fold) were significantly increased in both TGL and TGH compared with NTG mouse hearts (Fig. 3A-F). Moreover, reactive oxygen species (ROS) levels were significantly decreased in 6month-old TG mice (Supplementary Fig. S1), indicating diminished ROS signaling and redox imbalance. Concomitantly, significant increases in enzymatic capacities of key antioxidants (GCL: 3.5-fold, NQO1: 4.2-fold, GSR: 2.8fold, and CAT: 1.6-fold vs. NTG) were also observed in TGH mice (Fig. 3I-N). This strong correlation between the majority of antioxidant enzyme transcripts, protein abundance, enzyme activities, and total antioxidant capacity (TAC) $\left(r^{2}=0.876-0.964 ; p<0.05\right.$; Fig. $\left.3 \mathrm{G}, \mathrm{H}\right)$, along with blunted ROS in TG hearts, establishes the reliability of the model in regulating antioxidant pathways and inducing chronic RS.

\section{Chronic $R S$ causes maladaptive cardiac remodeling and reduces mouse life span}

We next evaluated the impact of RS on survival and morphological and biochemical changes in mouse hearts.

FIG. 3. Transgenic expression of caNrf2-TG induces proteins and antioxidant activity in mouse myocardium. (A-C) Representative immunoblots (IB) for myocardial antioxidant protein expression in cytosolic extracts from NTG and TG mice at 6-8 months. Each lane indicates an individual mouse. (D-F) Bar graphs illustrate the densitometry analysis performed using NIH ImageJ software of the Western blot data in panels (A-C), respectively. Data are plotted as relative density units of respective proteins after normalization to GAPDH density units. Raw images (uncut) are represented in Supplementary Fig S6. (G) Total antioxidant capacity was measured in NTG and TG mice at 6-8 months of age and represented as fold change $(n=6-8)$. (H) Analyses between the antioxidant protein abundance and total myocardial antioxidant capacity indicating a direct correlation. (I-N) Major antioxidant enzyme activities in NTG and TG mice at 6-8 months of age $(n=6-8)$. Data are represented as mean \pm SEM. Significance: $* p<0.05 ; * * p<0.01 ; * * * p<0.001 ; n s$, no significance. CAT, catalase; GAPDH, glyceraldehyde 3-phosphate dehydrogenase; GCLC, glutamate-cysteine ligase catalytic subunit; GCLM, glutamate-cysteine ligase modifier subunit; GPX1, glutathione peroxidase 1; GSR, glutathione reductase; GST- $\mu$, glutathione- $S$-transferase Mu (class); NQO1, NAD(P)H:Quinone Oxidoreductase 1; SOD1/SOD2, superoxide dismutase $1 / 2$. Color images are available online. 
A

B

CAT(59.8)

GPX1(24)

SOD1(18)

GAPDH(35.8)

\section{C}

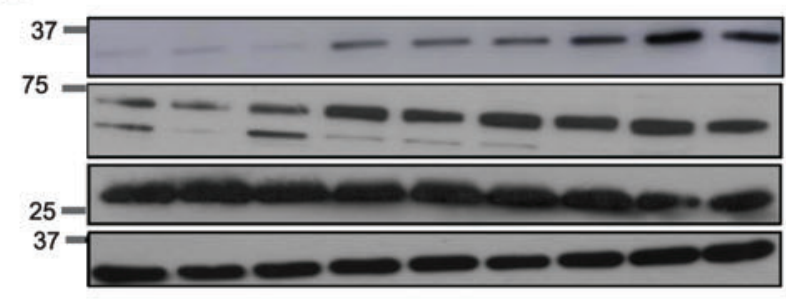

GCLM(31)

GCLC(72.6)

SOD2(26)

GAPDH(35.8)
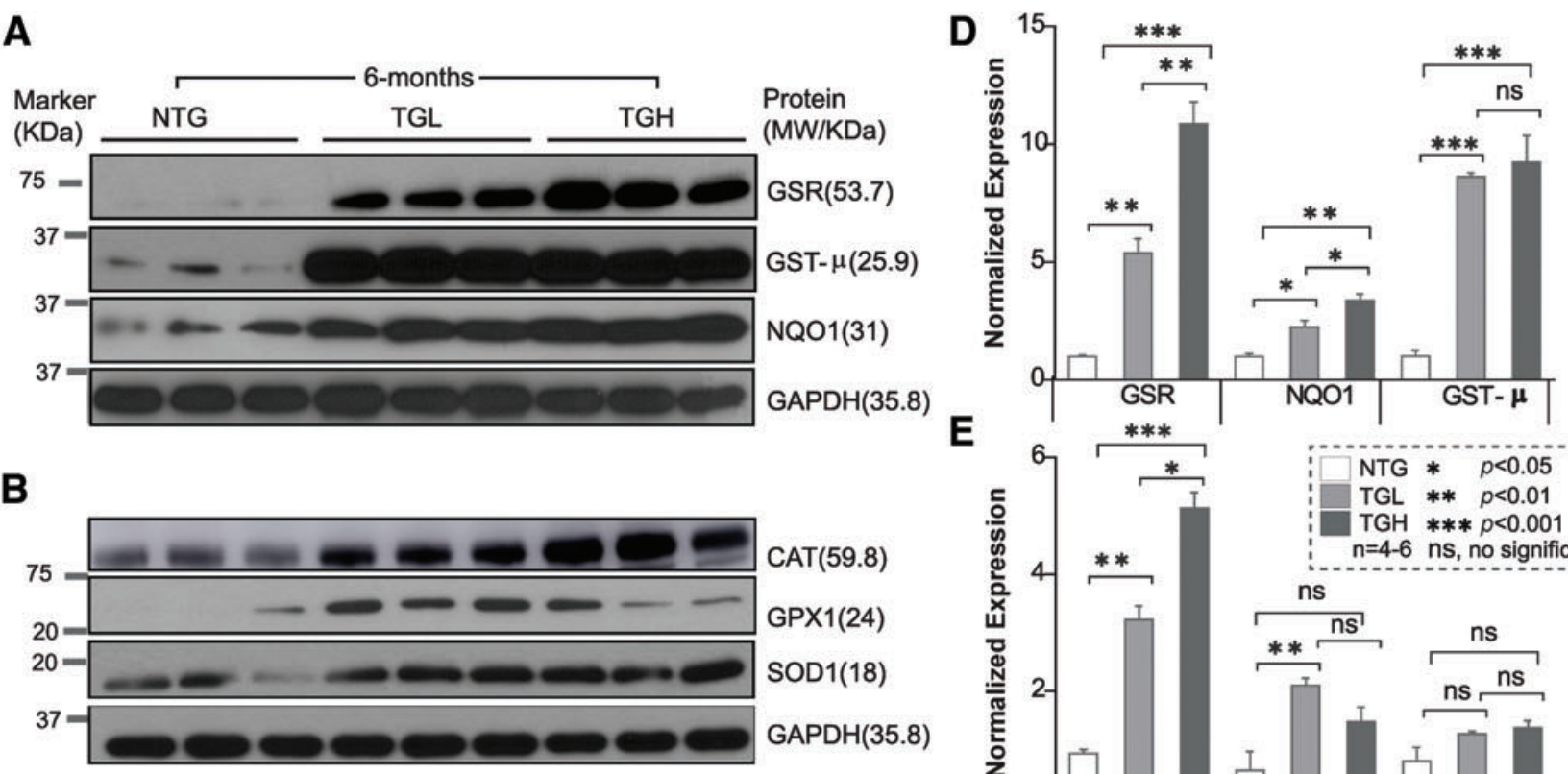

E

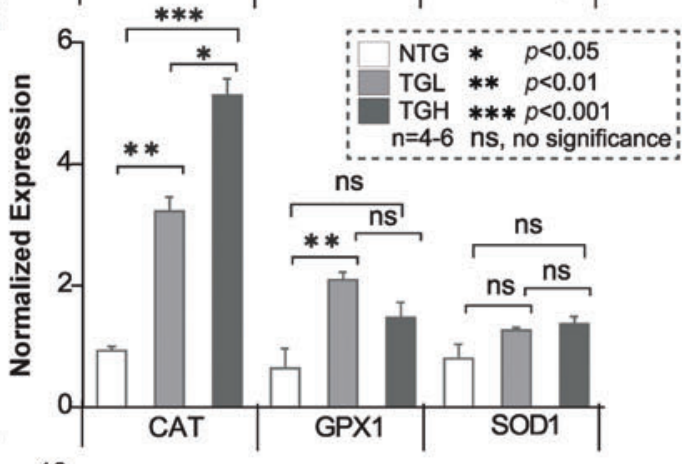

F

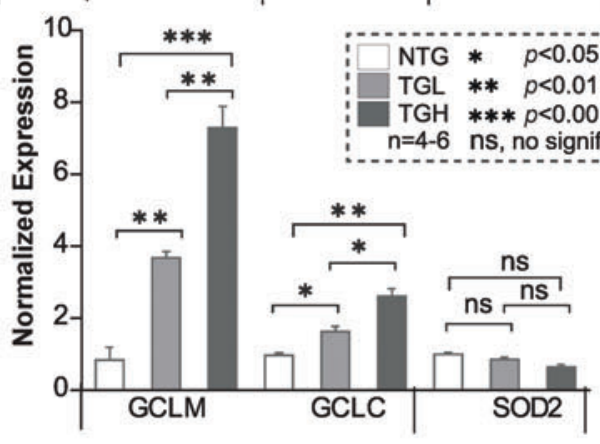

G

H

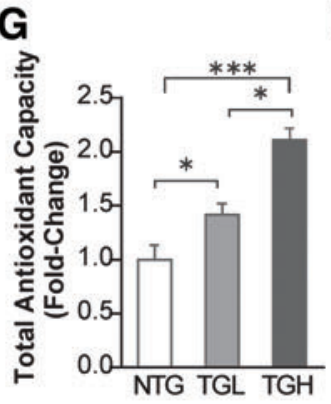

ᄂ 6-months -

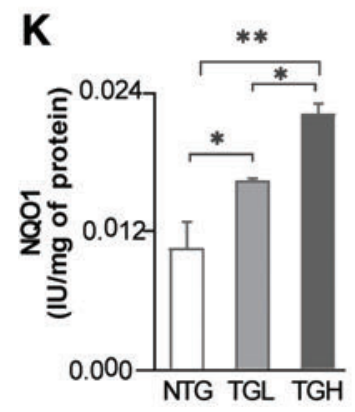

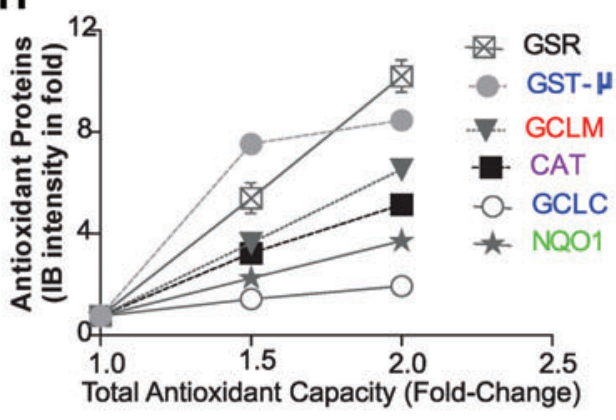

L

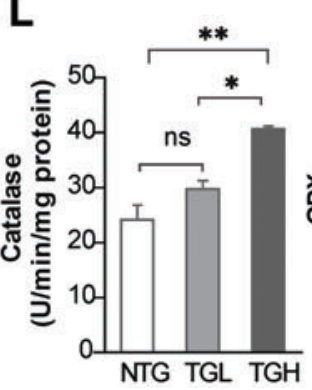

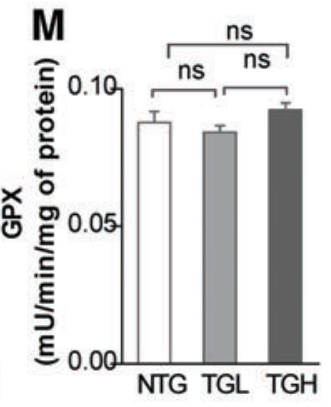

I
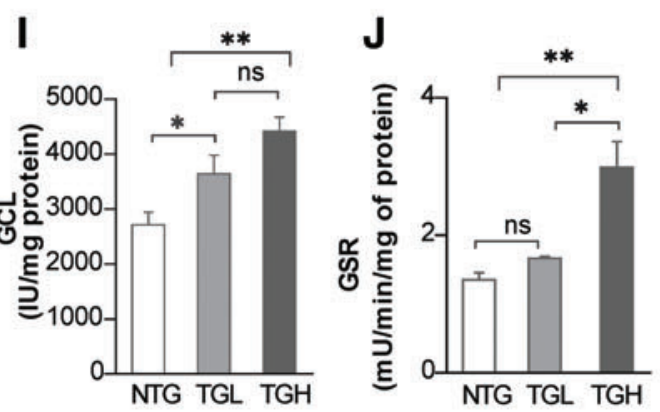

$\mathbf{N}$

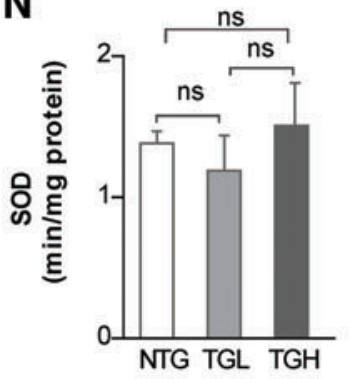

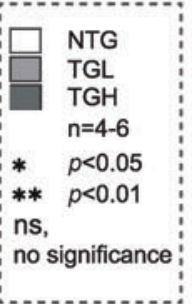



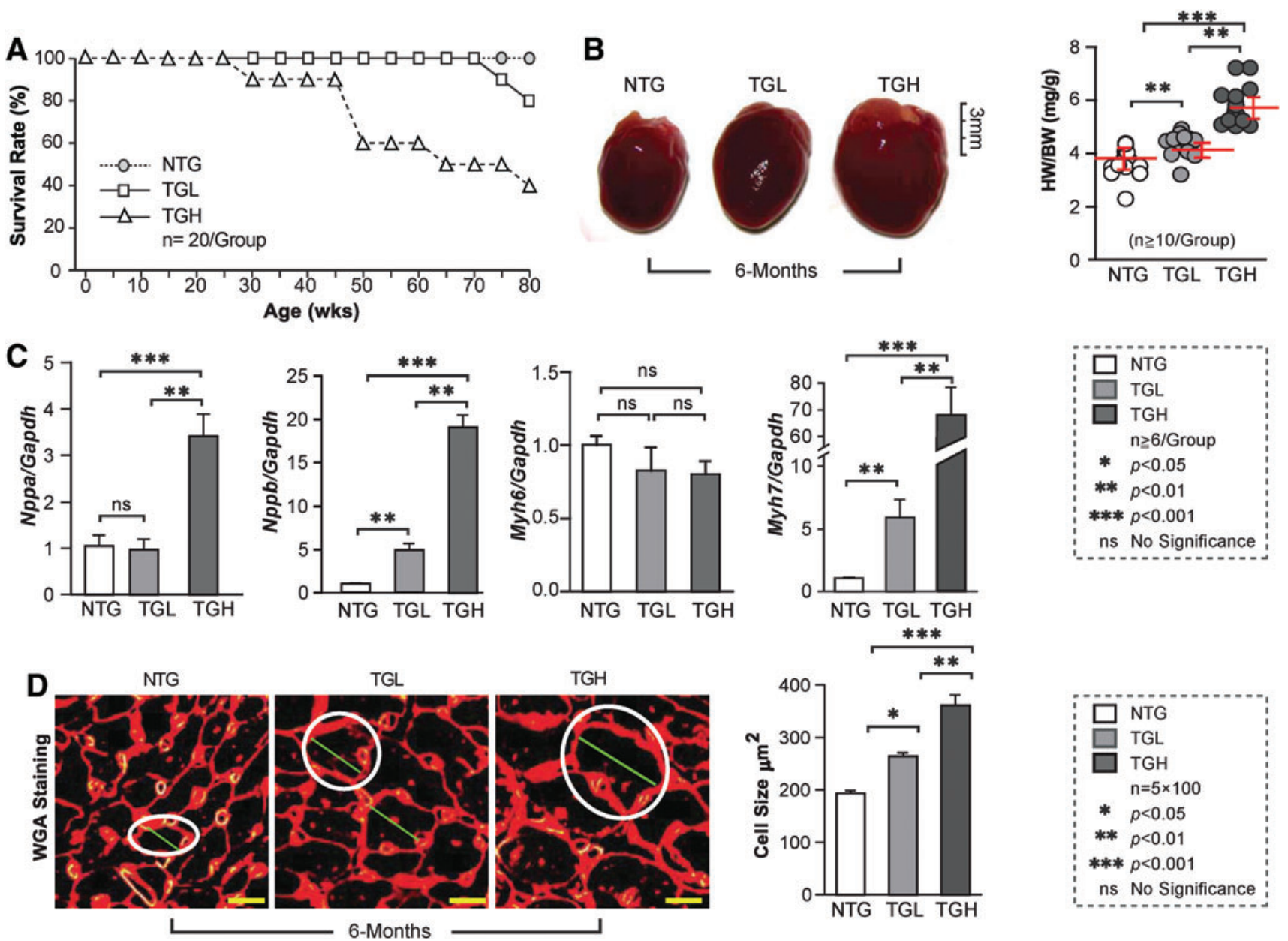

FIG. 4. cRS causes pathological hypertrophy in TG mouse hearts. (A) RS impact on mouse survival was determined by Kaplan/Meier curves for the 3 mouse groups ( $n=20 /$ group). (B) Representative images of hearts are shown for NTG, TGL, and TGH mice at 6 months of age. The heart-to-body weight ratios (HW/BW) were measured for NTG and TG mice at 6 months of age ( $n=10$ /group). (C) The transcript levels of cardiac hypertrophy markers (Nppa, Nppb, Myh6, and Myh7) were measured by qPCR among the three mouse groups $(n=6 /$ group) and normalized against Gapdh. (D) Representative histological images (cross section) of hearts are shown for NTG and TG animals at 6 months of age. Sample sections were stained with WGA. Cardiomyocyte size was determined in at least 100 cells/heart ( $n=5$ mice/group). The white circles indicate the size of the cells in different mice (genotypes). Data are represented as mean \pm SEM. Significance: $* p<0.05$; $* * p<0.01 ; * * * p<0.001$; ns, no significance. WGA, wheat germ agglutinin. Color images are available online.

Survival of TGL decreased by $20 \%$ at 18 months ( 80 weeks), compared with NTG mice. Most notably, by $\sim 11$ months $(50$ weeks) of age, $\sim 40 \%$ of TGH animals had died (Fig. 4A). While no effects were observed in 3-month-old mice (Supplementary Fig. S2), progressive increases in cardiac enlargement and heart weight/body weight ratio (HW/BW) were evident in TGH at 6 months of age (Fig. 4B). Transcripts of hypertrophic markers, including ANF (Nppa), BNF $(N p p b)$, and $\beta$-MHC $(M y h 7)$, were significantly increased in TGL or TGH 6-month mouse hearts (Figs. 2B, 4C) along with a decrease in $\alpha$-MHC (Myh6). Wheat germ agglutinin (WGA) staining demonstrated increased cardiomyocyte dimensions

FIG. 5. Chronic RS promotes hypersystolic function in myocardium of TG-mice. (A) Echocardiographic images (Mmode) were obtained using a high-resolution $(38 \mathrm{MHz})$ ultrasound to evaluate cardiac function. The LV cavity and wall thickness were measured in diastole (solid yellow line) and systole (dashed yellow line) for NTG and caNrf2 TGL and TGH mice at 6-7 months of age ( $n=8-10$ /group). (B, C) Quantitative analysis of echocardiograms for systolic function (EF) and hypertrophy. EDLVM was measured from 2D imaging, and longitudinal strain was obtained by M-mode imaging. (D) Representative images (B-mode) were captured in diastole of NTG, TGL, and TGH mouse hearts. The blue line represents the area distance within posterior inner wall. (E) Diastolic MV motions of the E wave (MV E) and A wave (MV A) were recorded using a tissue-Doppler instrument for NTG, TGL, and TGH mice. The yellow line represents distance (mm) movement of the annulus toward the left atrium in seconds during early/late filling. (F) The parameters of MV motion (E/A, $\mathrm{E}$ and A) demonstrate significant diastolic dysfunction in TGH mice at $\sim 6$ months of age $(n=8-10 /$ group). Data are represented as mean \pm SEM. Significance: ${ }^{*} p<0.05$; $* *<0.01$; $* * *<<0.001$; ns, no significance. EDLVM, end-diastolic LV mass; LV, left ventricle; MV, mitral valve. Color images are available online. 
A

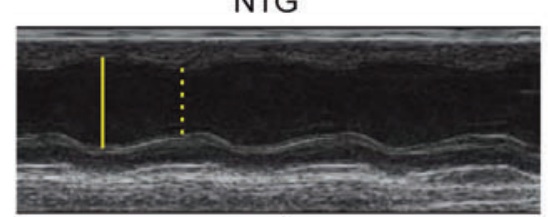

TGL

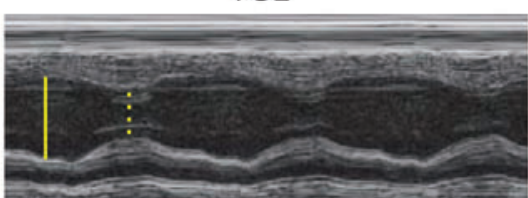

6-Months
TGH

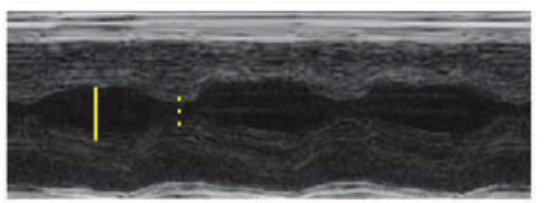

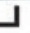

B
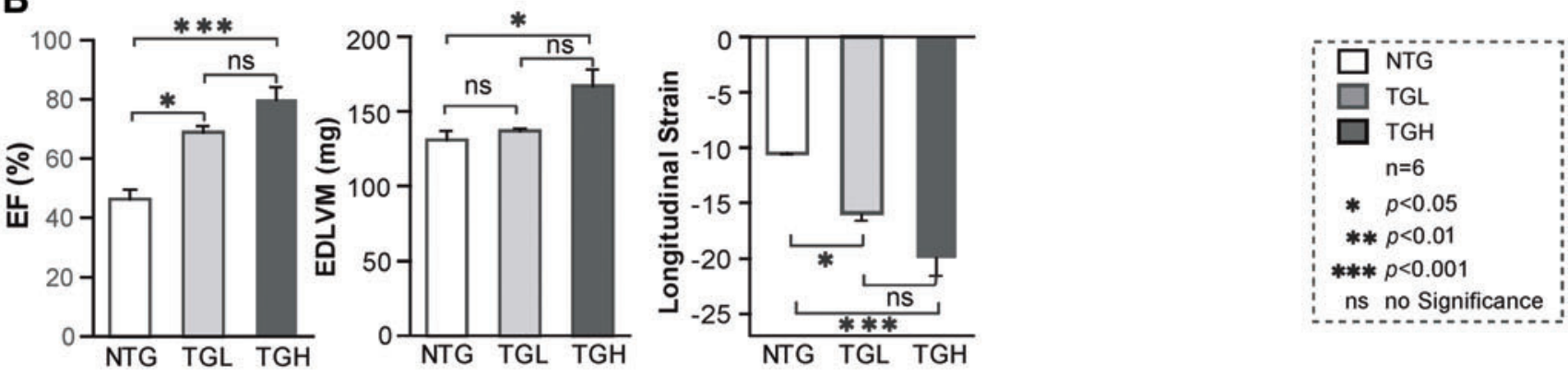

C
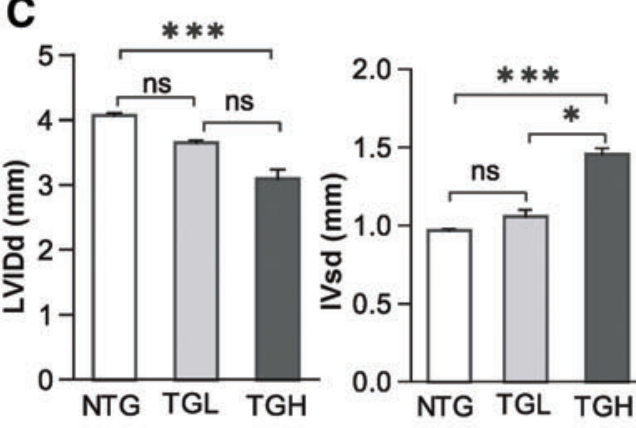

NTG
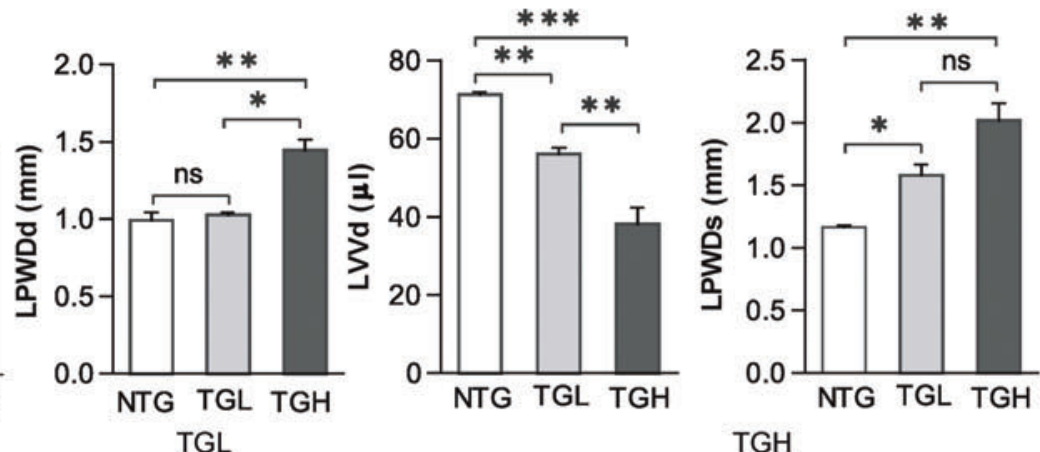

D
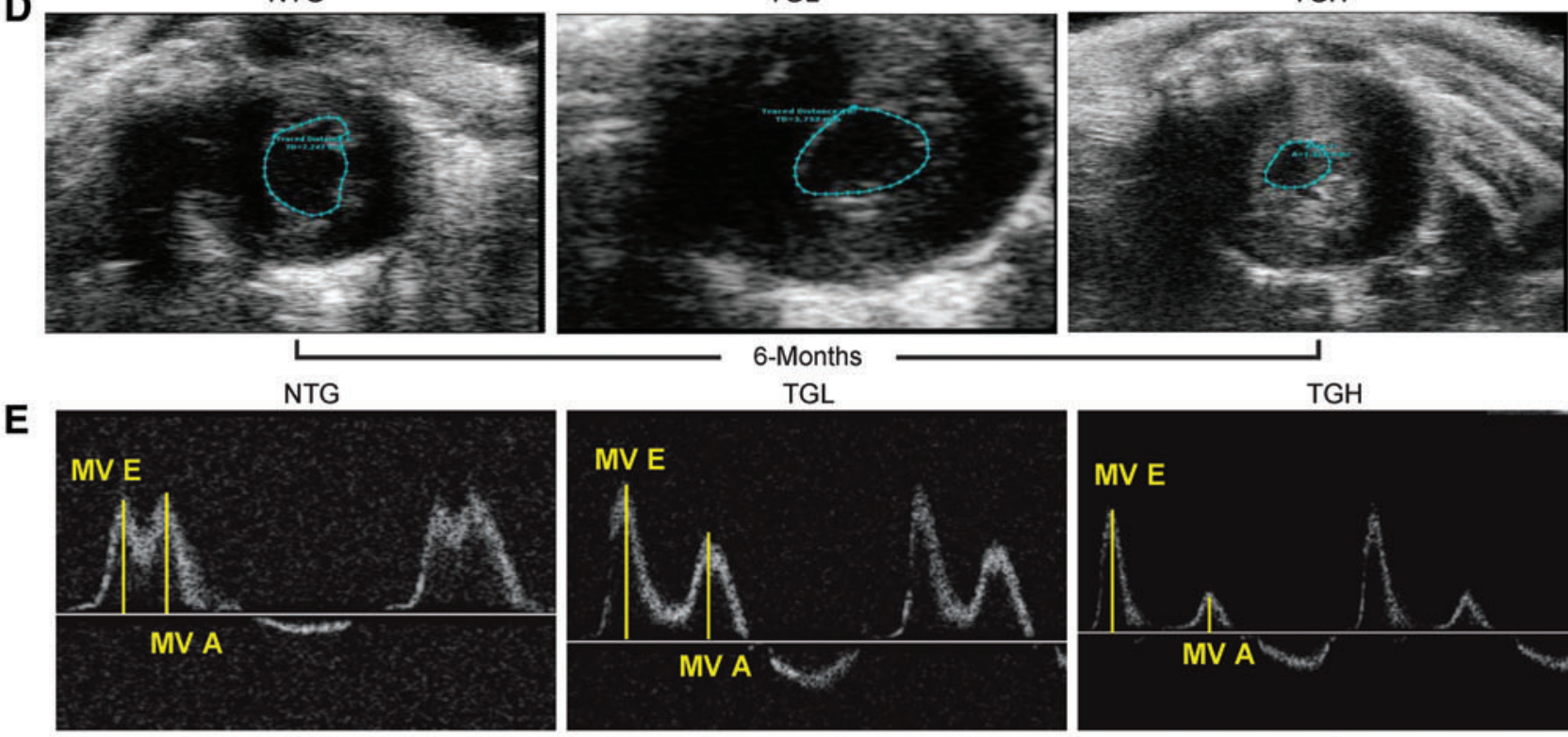

TGH
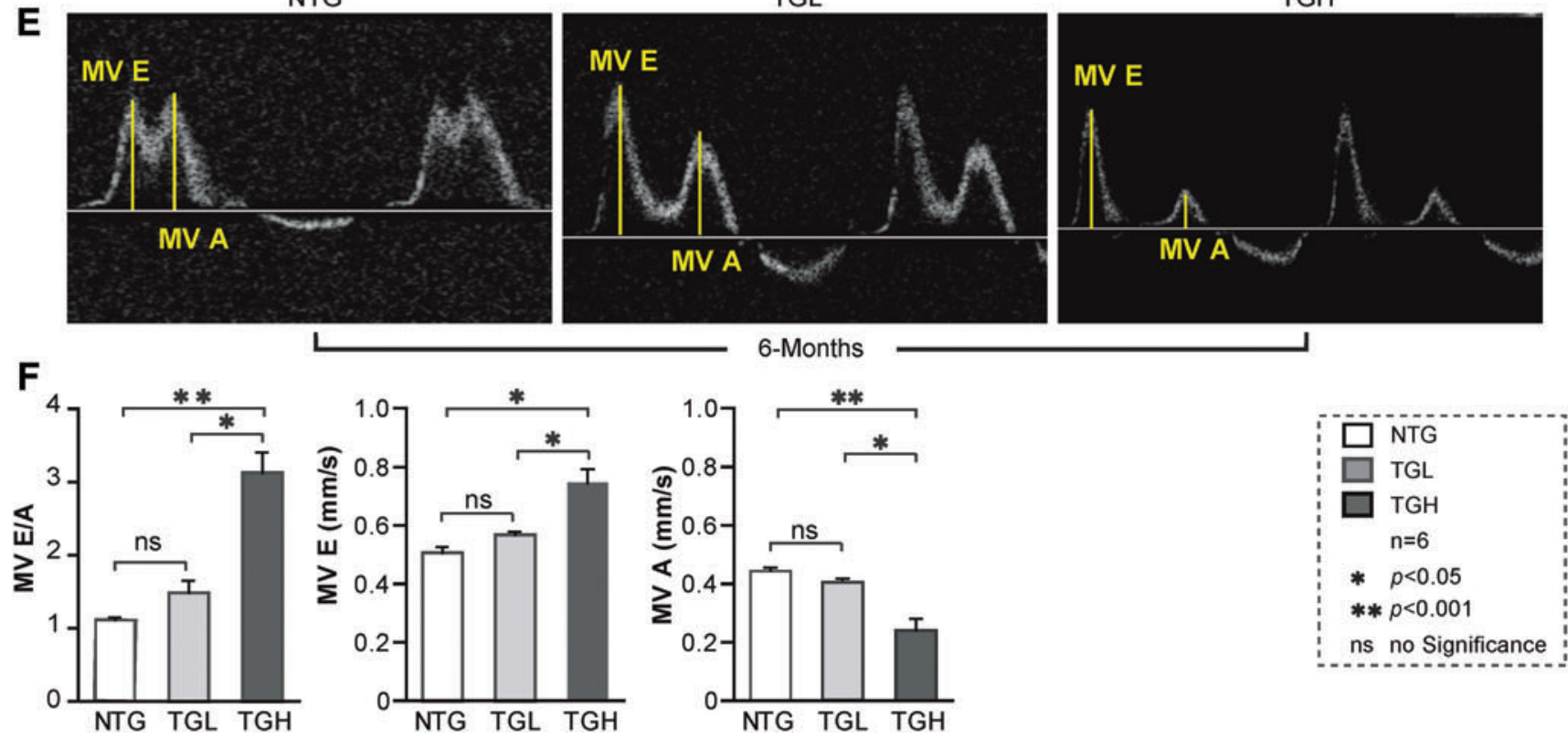
in TGL and TGH mice ( $+40 \%$ and $+100 \%$, respectively) at 6 months of age, providing further evidence for hypertrophy of cardiomyocytes (Fig. 4D).

\section{Chronic RS causes HCM with increased ejection fraction and diastolic dysfunction}

To assess the impact of chronic RS on cardiac function, we performed noninvasive measurements of cardiac function and hemodynamics on the NTG and TG mice. Representative echocardiograms (M-mode) obtained in the parasternal short-axis view showed deterioration in cardiac structure and function in TGH mice at $\geq 6$ months of age (Fig. 5A). In TGH, but not TGL hearts, significant concentric LV hypertrophy, decreased chamber volume, and increased cardiac contractility were observed. Compared with NTG mice, only systolic LV posterior wall thickness (LVPWs) increased in TGL mice, whereas both diastolic and systolic LV posterior wall thickness (LVPWd and LVPWs) increased in TGH hearts (Fig. 5C). End-diastolic LV diameter (LVIDd) displayed a significant reduction in TGH, but not TGL mice. Progressive decrease in end-diastolic LV volume (LVVd) was noted in TG mice (Fig. 5B), while the mean end-diastolic interventricular septal thickness (IVSd) increased by $\sim 50 \%$ in TGH mice. Short-axis echocardiography videos also demonstrated hypertrophic with hypercontractility in TGH hearts (Supplementary Video S1).

In parallel to echocardiography, we conducted strain analyses to obtain parasternal long-axis images. At $\leq 3$ months of age, changes in cardiac structure were less pronounced without major functional defects (Supplementary Fig. S3). EF was moderately or markedly increased in TGL or TGH mice, respectively, as were end-diastolic LV mass and longitudinal strain. Collectively, these results indicate a hyperdynamic state in TG mouse hearts (Fig. 5B). Short-axis frozen images illustrate LV hypercontractility and a closer proximity of posterior wall and septal motions during systole in TG mice (Fig. 5D), which may result in a delayed ventricular relaxation leading to progressive diastolic dysfunction.

We also conducted pulse wave/color Doppler analysis on the three mouse groups. LV diastolic function was determined by characterizing mitral valve (MV) motion. While there was a moderate change in the diastolic function at 3 months of age (Supplementary Fig. S3), by 6 months of age, TGH mice had developed overt LV diastolic dysfunction, as indicated by increased mitral inflow velocity (MV E/A ratio) and early diastolic flow velocity (MV E), as well as decreased MV late diastolic flow velocity (MV A) (Fig. 5E, F). This progressive structural remodeling (i.e., concentric hypertrophy) appeared to be coupled with gradual diastolic dysfunction and restrictive filling in TG mouse hearts. Also, at 6 months of age, diastolic dysfunction was much more severe in TGH than TGL mice. The results indicate that chronic RS causes dose-dependent HCM with impaired ventricular diastolic relaxation.

\section{caNrf2-TGL mice with moderate levels of Nrf2 activation increase $R S$-induced risks of maladaptive cardiac remodeling over time}

We next assessed whether sustained proreductive (PR) conditions promote cardiac remodeling over time (or aging) in TGL mice. To pursue this, we conducted echocardiography on TGL mice at $>15$ months of age (Fig. 6A-C). Compared with NTG controls, EF, LV mass, and IVSd were significantly increased, whereas the LVIDd and LVVd were notably reduced. Profound diastolic dysfunction was apparent by LV hypercontractility and abnormal MV motion, including an enhanced MV E/A ratio (Supplementary Video S2 and Fig. 6D, E). This suggests that even moderate RS may lead to substantial damage to the heart following prolonged exposure. In addition, the redox state (GSH/GSSH ratio) of the 15-month-old TGL mice was greatly increased and was comparable with the 6-month-old TGH mice (Fig. 6F). Furthermore, immunoblotting (IB) showed excessive antioxidant augmentation (GCLC, GCLM, GSR, NQO1) at >15 months of TGL compared with 6 months of TGH mice (Fig. 6G-F), with decreased ROS signaling suggesting that a chronic low dose of antioxidant accumulation also causes pathological cardiac diastolic dysfunction over time.

\section{Hyper-RS accelerates cardiac remodeling and dysfunction in double transgenic mice}

Because caNrf2-TGH mice exhibited hypertrophic remodeling and diastolic dysfunction at 6 months of age, but no obvious phenotype at 3 months, we questioned whether robust activation of Nrf 2 signaling actually provided the basis for this phenotypic switch. To address this issue, we created an even more severe RS model by crossing the caNrf2-TG with mice expressing a human Nrf2 (hNrf2) transgene (Supplementary Fig. S4A) and tested whether the cardiac remodeling was

FIG. 6. Echocardiographic analysis and antioxidant redox measurement of TGL mice at 15 months of age. (A) Representative M-mode images show significant increase in wall thickness of caNrf2-TGL mice at 15 months of age. The size of the LV cavity is described in yellow line during diastole (solid) and systole (dashed). (B) Echocardiographic analysis showed increased EF, left ventricular mass, LVIDd, LVVd, and IVSd for impaired systolic function at 15 months of caNrf2TGL mice. (C) Representative B-mode images for contraction in diastole and systole showed a high close proximity of the septum and posterior wall, which is comparable with 6-month-old TGH mice. The area within the posterior inner wall is captured in B-mode short axis and labeled in blue color. (D) MV motions were captured using a pulse wave color Doppler. The distance moved ( $\mathrm{mm}$ ) of the annulus toward the left atrium in seconds during early/late filling labeled is in yellow lines. (E) MV E/A and MV E values were increased, whereas the MV A was decreased, showing an increased diastolic dysfunction in TGL mice at the age of 15 months for diastolic dysfunction ( $n=6 /$ group). (F) Myocardial redox state (GSH/ GSSG ratio) was determined by enzyme-kinetic assays. (G) Immunoblots using heart cytosolic extracts from NTG, TGL (15 months), and TGH mice (6-8 months) for antioxidant proteins. Each lane represents individual mouse samples. Raw images (uncut) are represented in Supplementary Fig. S7. (H) DHE fluorescence-stained images for oxidative profiling in NTG, TGL (15 months), and TGH mice (6-8 months). Data are represented as mean \pm SEM. Significance: ${ }^{*} p<0.05$; $* * p<0.01$; $* * * p<0.001$; ns, no significance. DHE, dihydroethidium; EF, ejection fraction; IVSd, end-diastolic interventricular septal thickness; LVVd, end-diastolic LV volume. Color images are available online. 

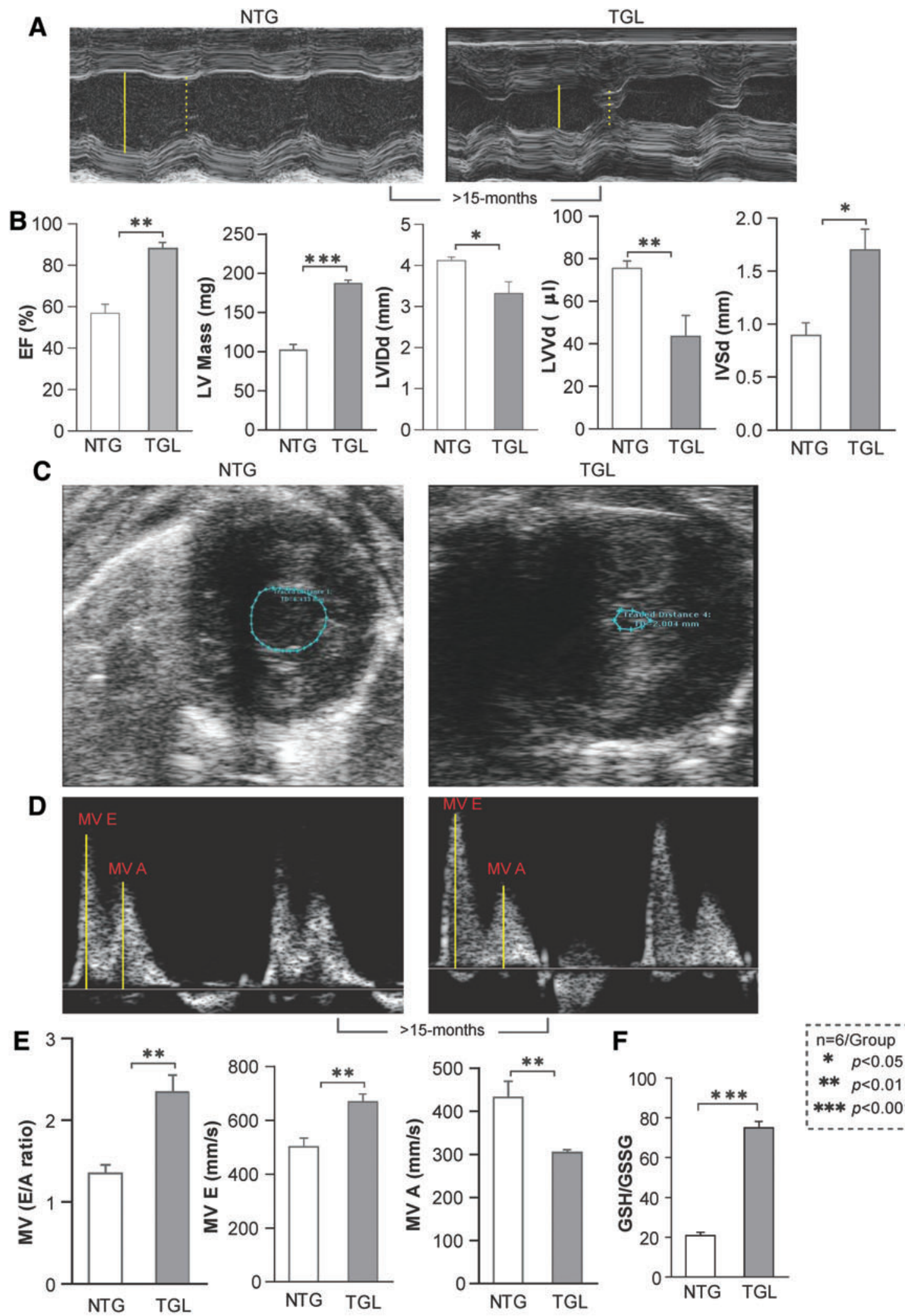

G NTG TGL(15M) TGH (6M)

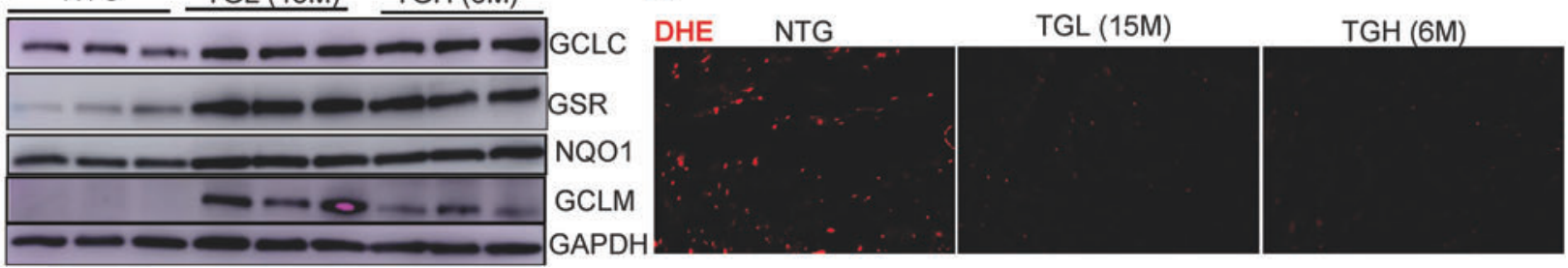


accelerated earlier than 6 months in the model. We performed qPCR for antioxidant and fetal gene expression, echocardiography for systolic and diastolic function, and immunofluorescence for GSH and 5,5-dimethyl-1-pyrroline $N$-oxide (DMPO) adducts. Results demonstrated significant increases in antioxidant genes (Supplementary Fig. S4B) with augmentation of GSH-N-Ethylmaleimide (Supplementary Fig. S4C) and decreased DMPO signals (Supplementary Fig. S4D) in double transgenic (DTG) mice, indicating that the hyper-reductive condition reduced the level of ROS.

Furthermore, by 2 months of age, DTG mice developed increased HW/BW ratio (Supplementary Fig. S4E) and increased hypertrophic markers (Supplementary Fig. S4F). In addition, echocardiography showed an increased EF (Supplementary Fig. S4G, H), with an increase in posterior septal wall (LWPD d) and septal wall diastole (IVS d) indicating that structural remodeling was evident in DTG mice by 2 months of age (Supplementary Video S3). Importantly, pulse wave Doppler analysis in the DTG mice (Supplementary Fig. S4I-J) showed increased inflow MV E/A ratio compared with the NTG littermates. Furthermore, DTG mice exhibited a higher amount of antioxidant protein expression compared with caNrf2-TGH mice at $\sim 2$ months of age (Supplementary Figs. S4K, S9), indicating that the hyper-reductive condition led to HCM with restricted diastolic filling by 2 months of age.

\section{Buthionine sulfoximine-mediated GSH depletion prevents $R S$ and delays onset of cardiac remodeling in $\mathrm{TGH}$ mice}

As the most abundant nonprotein thiol source, the cellular GSH pool accounts for most of the reducing power accompanying Nrf2 activation. To restore redox balance in RS hearts, we treated TGH mice with BSO in vivo $(33,35)$ (Fig. 7A). Early BSO intervention (starting at 1.5 months of age and up to 4.5 months) successfully depleted GSH and shifted redox status back to an NTG level (Fig. 7B). Of note, BSO treatment also prevented cardiac hypertrophy, as demonstrated by $\mathrm{HW} / \mathrm{BW}$ ratio and hypertrophic marker levels (Fig. 7C, D), and significantly improved the diastolic and systolic function of TGH mice (Fig. 7E, F). Furthermore, no significant changes were noted in key antioxidant protein levels (GCLC, GST- $\mu$, and GCLM) (Fig. 7G). These results suggest that pharmacologic depletion of GSH can prevent RS and delay pathological remodeling of the myocardium.
During BSO treatment, we also observed that GSR levels were partially restored, suggesting that regulation of some antioxidant enzymes may be sensitive to redox perturbation (GSH levels). It is also possible that their turnover might be regulated by mechanisms independent of Nrf2 levels. Concomitantly, BSO-treated mice showed moderate levels of ROS, confirmed by dihydroethidium (DHE) florescence staining (Fig. $7 \mathrm{H}$ ), suggesting that early BSO treatment rescues the myocardium from RS and restored the basal ROS signaling.

\section{Correlation analysis between different redox levels and its effects on systolic and diastolic function at different ages in Nrf2-transgenic myocardium}

Based on the changes in redox conditions associated with structural and functional remodeling in the myocardium of TG (caNrf2) at different ages (3, 6, and 15 months of age), and of DTG (hNrf2:caNrf2; 2 months) compared with NTG mice, we correlated the dose- and time-dependent impact of RS on the severity of myocardial dysfunction. Dose-dependent increases in reductive redox (TGL and TGH) proportionally increased systolic function of the heart at $3(8 \%$ and $41 \% \mathrm{vs}$. NTG) and 6 (50\% and 72\% vs. NTG) months. Of note, TGL mice at 15 months of age showed significantly greater changes in EF (69\%) compared with age-matched NTG mice. DTG mice at 2 months of age showed an increased EF (44\% vs. NTG), which was comparable with 6-month-old TGL mice (Fig. 8A).

Analysis of diastolic function showed a dose-dependent increase in the MV E/A ratio in TGL and TGH mice $(10 \%$ and $27 \%$ vs. NTG) at 3 months of age, whereas chronic increases (adaptation) in MV E/A ratio of TGH (>100\% vs. NTG) mice at 6 months of age resulted in progressive diastolic dysfunction and HF. The TGL mice at 6 months of age showed less change in diastolic function (33\% vs. NTG), whereas at 15 months they developed a significant diastolic dysfunction (73\% vs. NTG). Surprisingly, DTG mice developed severe diastolic dysfunction ( $69 \%$ vs. NTG) by 2 months of age, which was comparable with the 15-month-old TGL mice. Compared with 3-month-old TGH or 6-month-old TGL mice, the degree of diastolic dysfunction was significantly higher in DTG mice at 2 months (Fig. 8B). We also compared the impact of systolic versus diastolic changes in the context of a reductive environment (Fig. 8C). The analysis demonstrated that increased EF augments MV $\mathrm{E} / \mathrm{A}$ ratios. However, chronic increase in both leads to an

FIG. 7. Early rescue of redox homeostasis in TGH mice prevents development of hypertrophic cardiomyopathy disease phenotype. (A) Schema of BSO administration (before the onset of cardiac remodeling, at $\sim 6$ weeks of age) to TGH mice. NTG mice were used as control. (B) Redox levels for glutathione (GSH and GSH/GSSG ratio) were determined by a kinetic spectrophotometric assay in TGH and TGH+BSO mice ( $N=5$ mice/group). (C) Morphologies of NTG, TGH, and TGH+BSO hearts and heart-to-body weight (HW/BW) ratios from autopsy confirm the absence of hypertrophy in TGH+BSO mouse hearts $(N=5-6$ mice from each group). (D) Real-time qPCR analyses of cardiac hypertrophy markers (Nppa and Myh7) in TGH+BSO mice are shown. (E) M-mode echocardiographic images with high-resolution (38 MHz) ultrasound were obtained to evaluate systolic function (EF) in TGH+BSO mice ( $n=5-6 /$ group). The LV cavity size is described in yellow line during diastole (solid) and systole (dashed). (F) MV (diastolic) function was measured by tissueDoppler to determine MV (E/A) of the three mouse groups. The distance moved $(\mathrm{mm})$ of the annulus toward the left atrium in seconds (s) during early/late filling is labeled in yellow lines. (G) Immunoblots of antioxidant enzymes in the three mouse groups were examined. BSO treatment did not affect the expression levels of GCLC, GCLM, and GST- $\mu$, whereas that of GSR was reduced, ( $n=3$ mice/group). Raw images (uncut) are represented in Supplementary Fig. S8. (H) Fluorescencebased oxidative profiling using DHE in myocardium of NTG, TGH, and BSO-treated TGH mice. Data are represented as mean \pm SEM. Significance: $*^{*}<0.05$; $* *<<0.01$; $* * * p<0.001$; ns, no significance. BSO, L-buthionine-SR-sulfoximine. Color images are available online. 
A

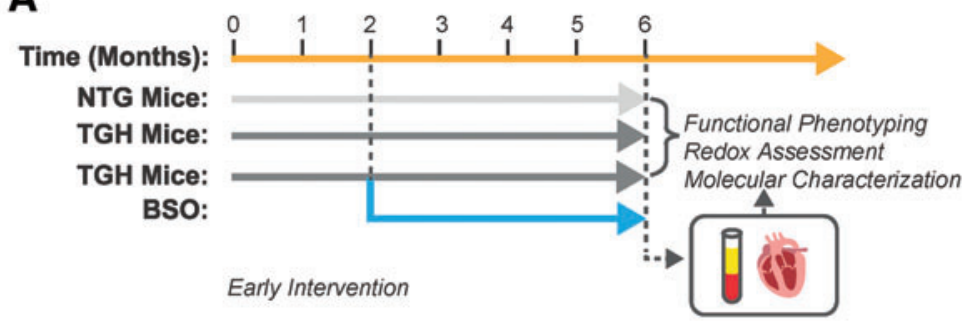

C

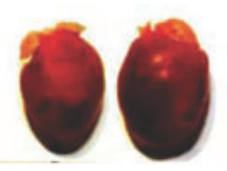

NTG TGH TGH+BSO

ᄂ 6-Months -

E
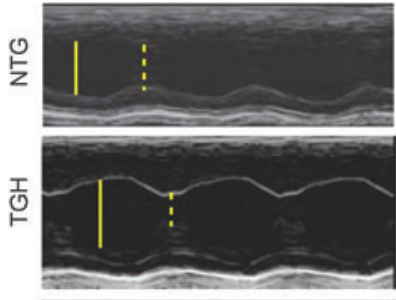

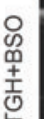

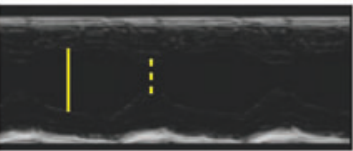

ᄂ-Months -
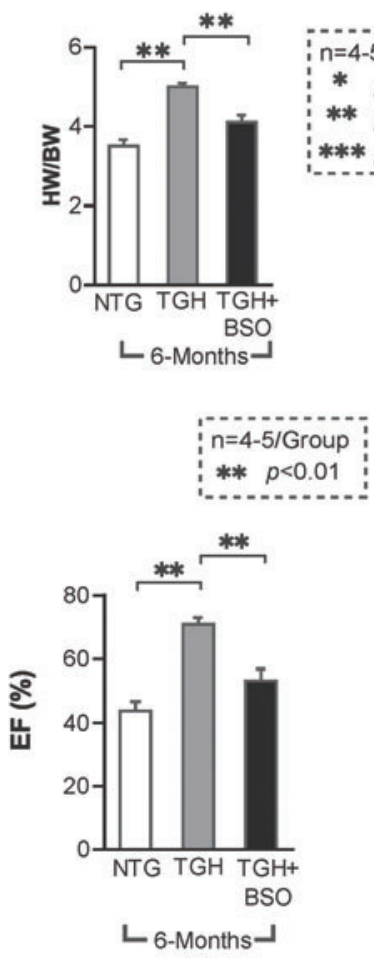
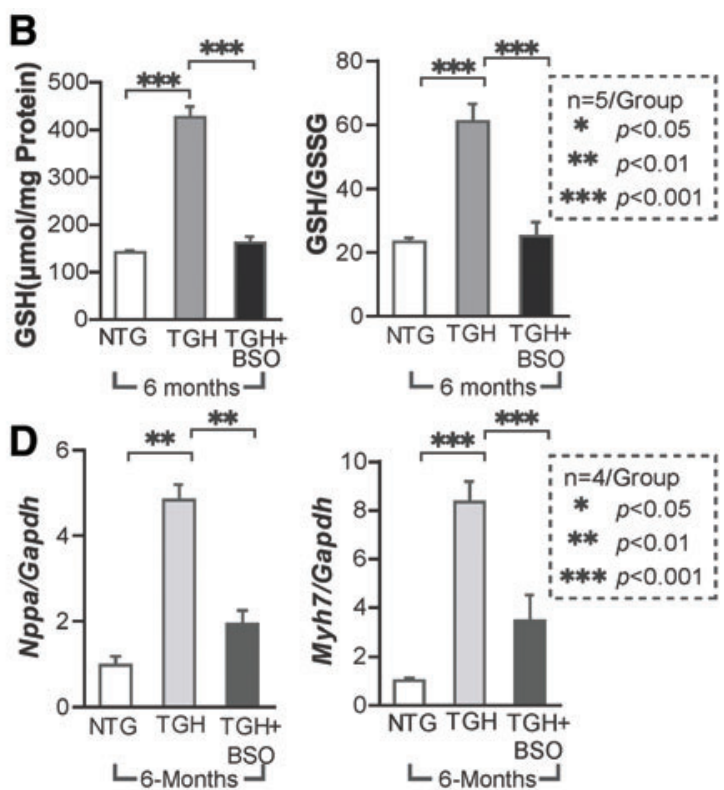
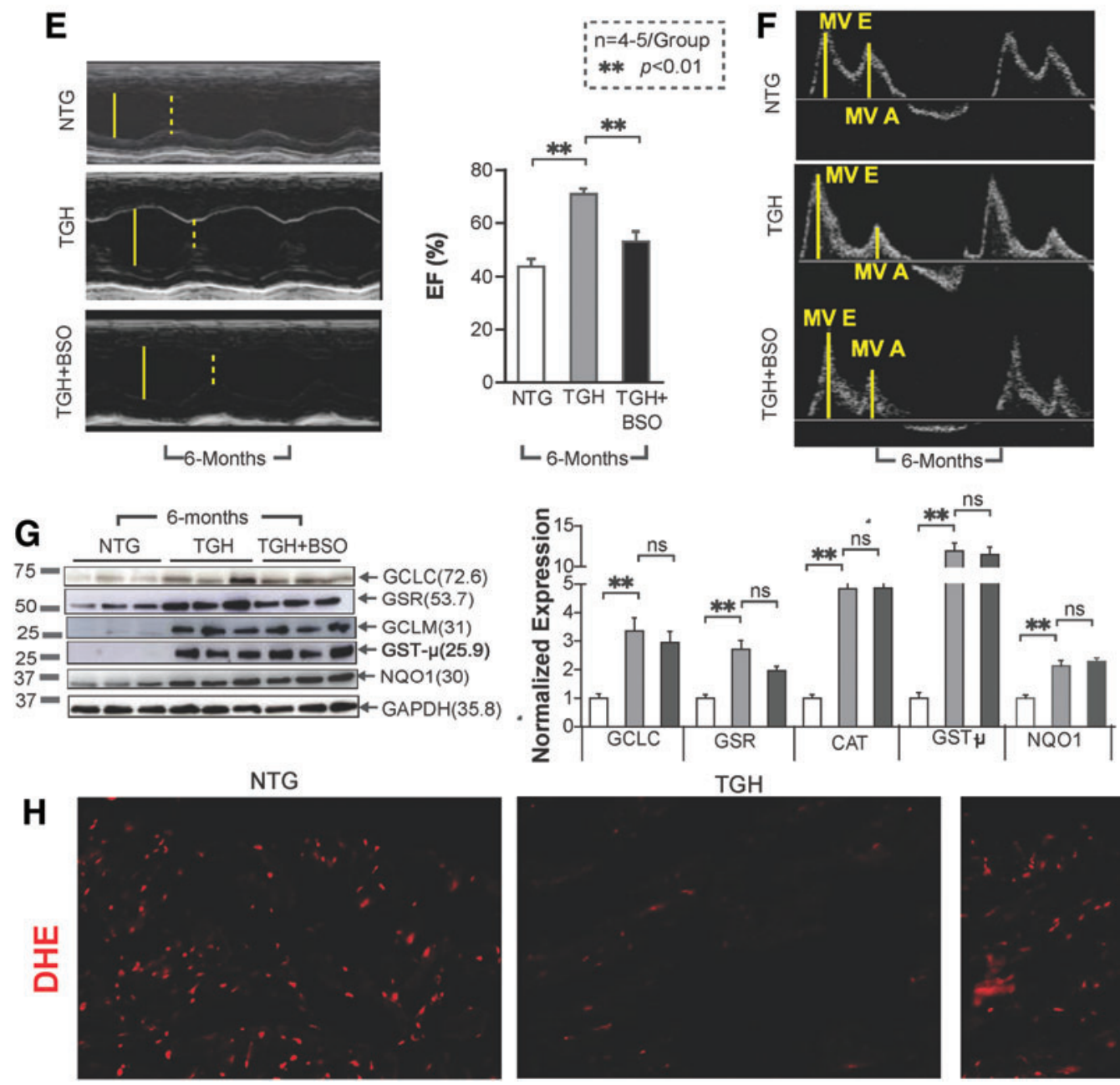

$\mathrm{n}=4-5 /$ Group ** $p<0.01$
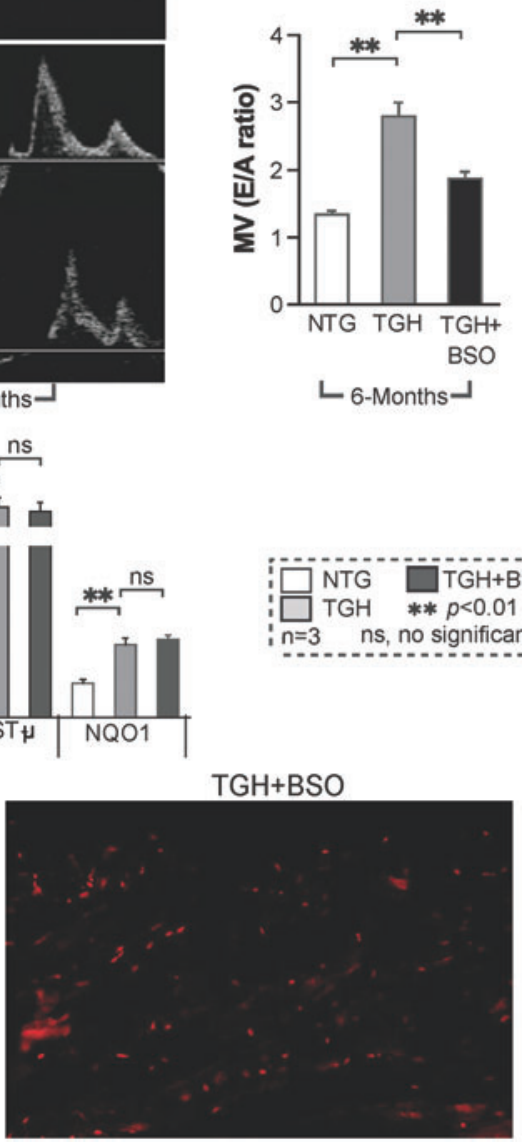
A

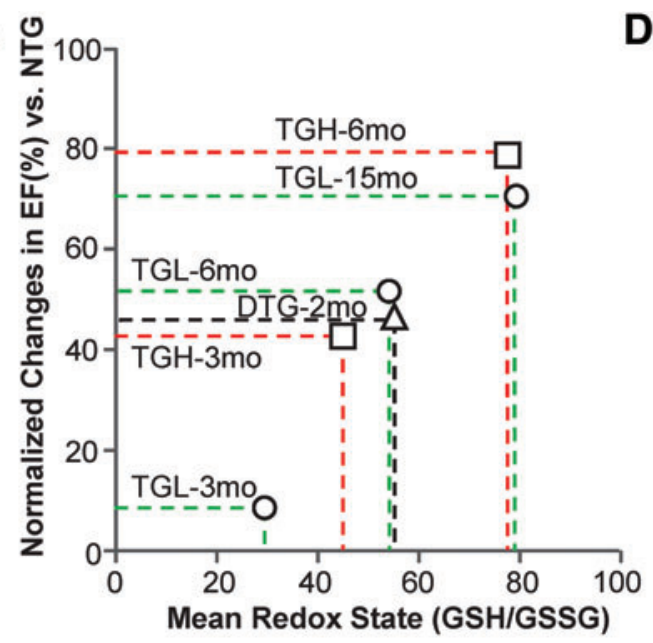

B

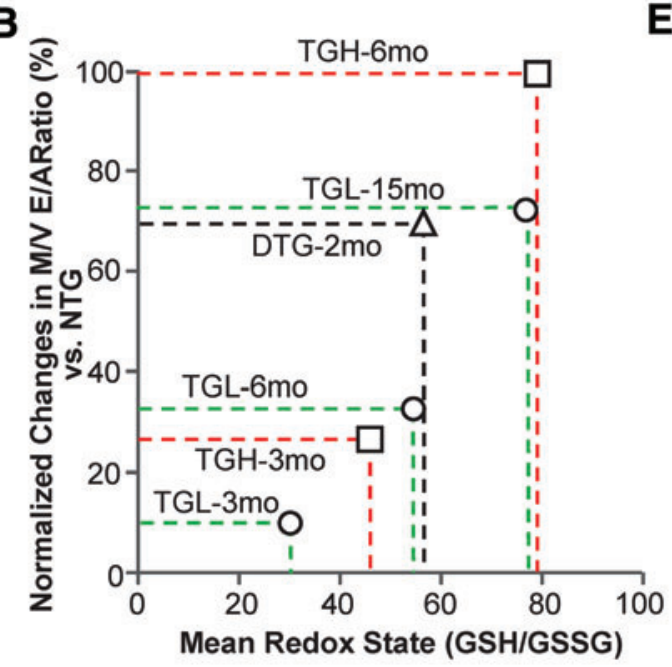

C

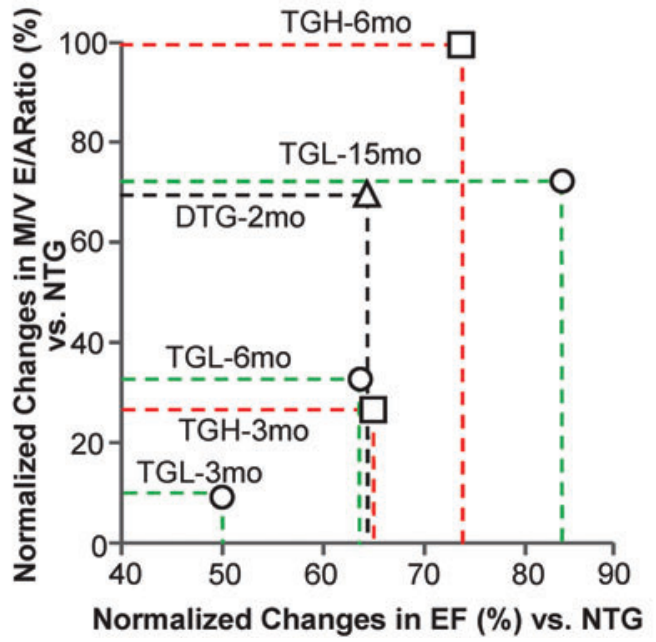

E
Redox, cardiac structural \& functional phenotype
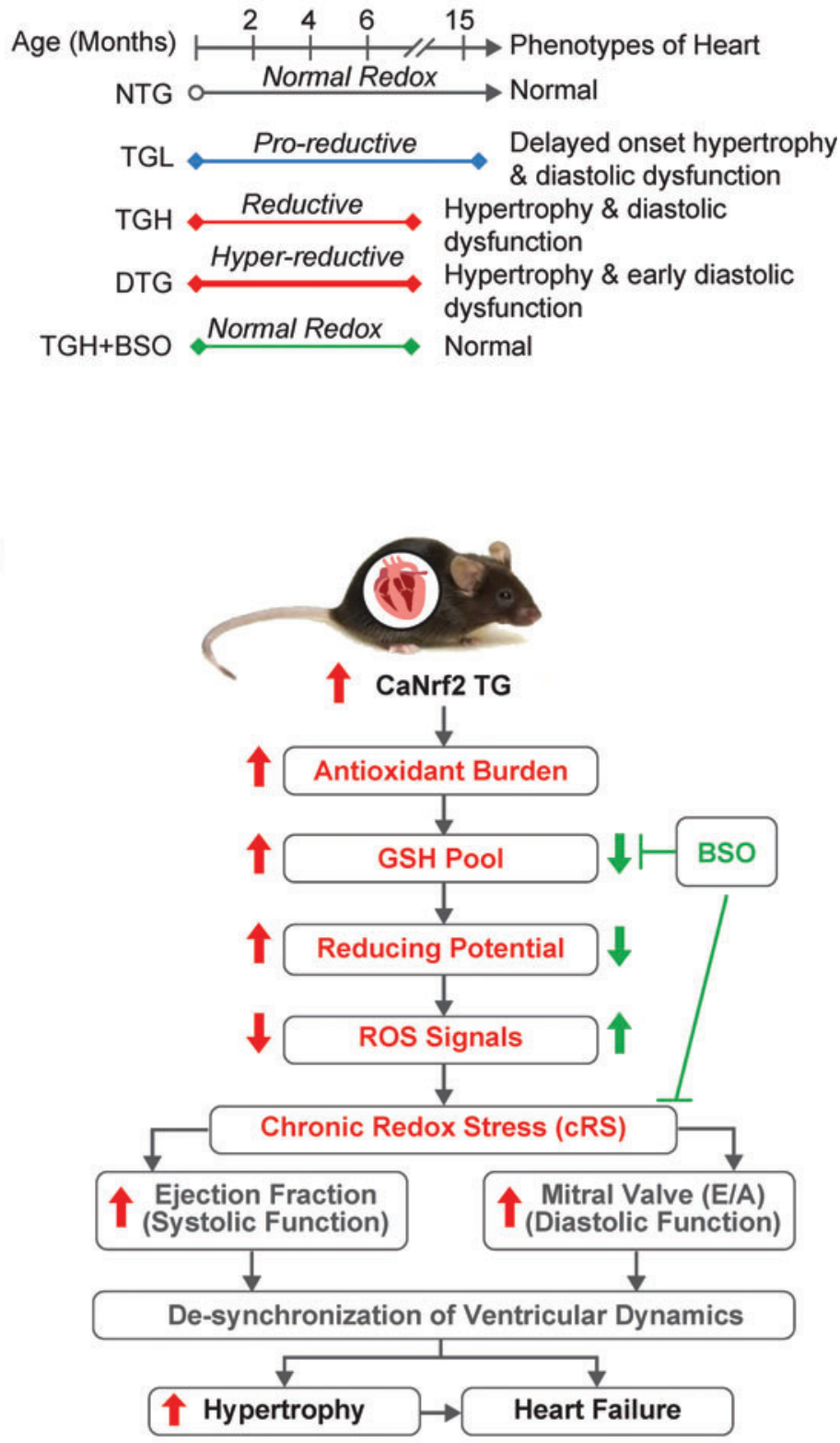

FIG. 8. Correlation between redox status and functional changes indicated a cRS condition induces pathologic cardiac remodeling. Correlations among redox status (dose and time dependent) and cardiac functional outcomes (systolic and diastolic) are demonstrated in both young (2,3, and 6 months) and old (15 months) TGL, TGH, and DTG mice, indicating remarkable impacts of cRS on both (A) systolic (i.e., EF\%) and (B) diastolic (i.e., M/V E/A ratio) functions. (C) The diastolic function was deteriorating to increase reductive potential, whereas systolic function was elevated, suggesting that (D) varying degrees of reductive-redox through dose- and time-dependent Nrf2 expression influence on the structural and functional phenotypes of heart. (E) A schematic overview of how the caNrf2 expression induces cRS and depletes biological ROS signals, as well as how cRS ( $>6$ months) induces the cardiac structural and functional remodeling by promoting cardiac hypertrophy and diastolic dysfunction. Doubling the myocardial Nrf2 levels (in DTG) further advances RS and accelerates cardiac remodeling/ diastolic dysfunction as early as 1.8 months of age. Pharmacologic depletion of glutathione using BSO abolishes RS-mediated myocardial remodeling. cRS, chronic reductive stress; ROS, reactive oxygen species. Color images are available online. 
abnormal increase in systolic function with impaired restrictive filling, which results in diastolic dysfunction during chronic RS (Fig. 8A-C).

\section{Discussion}

The pathological implications of oxidative stress in various cardiac disorders have been extensively investigated over the past six decades $(16,50,60)$. Exogenous supplementation of antioxidants and endogenous activation of antioxidant enzymes are the two major strategies used to curtail this oxidative damage. However, several antioxidant supplementbased interventions have failed to be beneficial $(2,4,9,15$, $30,59)$ and chronic infusion of antioxidants increased ischemia/reperfusion patient mortality $(3,6)$, leaving the activation of endogenous antioxidant genes a more desirable approach. While it has been assumed that a shift to more reductive redox conditions would be beneficial, it is possible that excessive reductive capacity could actually be harmful.

Single-nucleotide polymorphisms found in human gene disrupt Nrf2 protein expression and have been associated with a broad panel of human diseases (13). Especially, the newly discovered mutations (G31R, E79K, T80K, and G81S) in Neh2 domain (i.e., one of the Keap1-Nrf2 binding domains) led to an elevation of NRF2 level, overexpression in many antioxidant genes, and the manifestation of a multisystem disorder in four pediatric patients (21). Heart defects included thickened bicuspid aortic valve, atrial septal defect, and symptoms of cardiomyopathy. Notably, the level of plasma homocysteine, a redox marker, was reduced among these patients, suggesting that chronic activation of Nrf2 disrupted the redox balance and contributed to disease manifestation. However, the molecular mechanisms by which excessive redox shifts caused maladaptive cardiac remodeling remained largely unknown, partially due to a lack of appropriate animal models.

To investigate whether excessive antioxidant capacity leading to RS causes pathological changes in the heart, we developed a caNrf2 transgenic mouse model (53) with TGL and TGH mice mimicking PR and RS conditions, respectively. The model enables us to study the effects of different levels of RS on myocardial pathophysiology (Fig. 2). In addition, we developed a cardiac-specific DTG mouse expressing human Nrf2 with mouse caNrf2, which induces hyperRS, accelerated cardiac remodeling, and dysfunction.

A noteworthy finding of the current investigation is that PR conditions in TGL mice appear not to be harmful at a young age, whereas RS in young TGH mice induced pronounced pathological changes (Fig. 4). However, TGL mice did develop significant cardiac remodeling at an older age ( $>15$ months) (Fig. 6). Our data suggest that sustained antioxidant burden (e.g., GSH and antioxidant enzymes), even of relatively low intensity, but over a long period of time led to diastolic dysfunction in mice. To our knowledge, our models are the first transgene dose-dependent (i.e., TGL and TGH) RS models that developed age-dependent, pathological, structural, and functional remodeling of myocardium (Fig. 4).

Of note, mice with RS developed a distinctive phenotype, demonstrating HCM with abnormally increased ejection fraction $(\mathrm{HCM} i \mathrm{EF})$. Perhaps the model is an extreme example of reductive shift. In the clinical setting, RS and the corresponding phenotype might be less apparent, similar to the recent discoveries of HF phenotype with normal or preserved $\mathrm{EF}(\mathrm{HF} n \mathrm{EF}$ or $\mathrm{HFpEF})(5,7,65)$. Based on our finding of an association between redox conditions and disease phenotype, we suggest that HF subjects with systolic dysfunction are more likely to be under oxidative stress and sensitive to treatment with antioxidant supplements or diets enriched in antioxidants. In contrast, HF subjects with chronic hypertension might be under an RS and have increased risks when exposed to/treated with antioxidant drugs (56). Clinically, HFpEF is characterized by diastolic dysfunction $(36,39)$. Affected individuals tend to be elderly women with diabetes, hypertension, and coronary artery disease $(11,28)$. The TGH mice exhibited an abnormal increase of EF, developed cardiac hypertrophy, and increased passive myocardial stiffness without evidence of fibrosis. Together, these phenotypic properties suggest that excess antioxidants promote systolic function through progressively increased ventricular stiffness with prolonged relaxation, leading to diastolic dysfunction and HF (Fig. 5).

In the present investigation, we also demonstrated that pharmacological depletion of GSH rescued TG mice from adverse cardiac remodeling. In TGH mice that developed a severe disease phenotype by $\sim 6$ months, GSH depletion with BSO attenuated RS and prevented RS-induced pathological myocardial remodeling (Fig. 7). Our attempts to rescue the TG-mice from adverse cardiac remodeling through pharmacological inhibition of GSH are encouraging. BSO-mediated GSH depletion before developing cardiac hypertrophy (1.5 months of age) prevented RS and rescued the DTG from cardiac dysfunction.

In summary, harnessing the power of cardiac-specific constitutive Nrf2 overexpression in mouse model(s) (PR and RS in TGL and TGH, respectively) can help us understand the influence of antioxidant/redox manipulation on the development of cardiac pathology and disease. Interventions targeting RS at the different stages of disease progression (before and at the onset) may offer new approaches to preventing disease progression.

\section{Conclusions}

Short-term Nrf2 activation has demonstrated beneficial effects in different model systems, especially in an oxidative stress environment (32). We report that sustained or chronic Nrf2 activation results in RS, which leads to progressive pathological remodeling and HF through diastolic dysfunction. In the current investigation, we delineated the functional phenotypes and molecular characteristics of genetic models of RS. The results provide new insights by which to evaluate the impact of chronic antioxidant abundance in HF. Under chronic RS, mice developed $\mathrm{HCM} i \mathrm{EF}$, pointing to a potential direction for further investigation in HF subjects. Furthermore, our results show that, in addition to upregulation of genes involved in GSH metabolism, protein folding response also significantly disrupted under RS. Hence, we speculate that enhanced GSH accumulation might impair the protein folding mechanisms. However, it requires proteome-wide characterization for RS-induced changes in protein features (e.g., expression, turnover, and posttranslational modifications) to better comprehend the molecular interplays bridging excessive reductive shift and pathological remodeling of heart. 


\section{Detailed Methods}

Reagents

RNeasy kit (74106), QuantiTect SYBR Green PCR, and QuantiTect reverse transcription kit (205313; Qiagen) were purchased from Qiagen, Inc. (Valencia, CA). Secondary antibodies for immunofluorescence conjugated with Alexa Fluor 488/594 anti-rabbit (A11008/A11012) and anti-mouse (A21042/A11005) were obtained from Life Technologies Corporation (Carlsbad, CA). The anti-rabbit (PI-1000) or anti-mouse (PI-2000) secondary antibodies for immunoblots (horseradish peroxidase-conjugated with $\mathrm{IgG}$ ) were purchased from Vector Laboratories (Burlingame, CA). Primers for qPCR were designed using the Harvard Medical School PrimerBank website and purchased from Integrated DNA Technologies (IDT) (Coralville, IA). Protein Assay reagent (\#500-0006) was procured from Bio-Rad (Hercules, CA). The Trans-AM Nrf2 promoter binding assay kit (50296) was from Active Motif (Carlsbad, CA). All other chemicals, including bovine serum albumin (BSA), RNAlater, reduced and oxidized GSH as standards, were purchased from Sigma Aldrich unless otherwise stated.

\section{Animal models}

All animal experimental procedures were approved by the Institutional Animal Care and Use Committee (IACUC) of the University of Utah and University of Alabama at Birmingham. Two distinct transgenic mouse lines were used. The heartspecific caNrf2 transgenic model (TGL and TGH) has been described in our recent publication (53). The DTG mice were generated by crossing the caNrf2-TGH mice with hNrf2-TG mice. Briefly, to overexpress human Nrf2 in the myocardium, the Nrf 2 construct was cloned in a mouse $\alpha$-MHC promoter. The $\alpha$-MHC-hNrf2 fragment was injected through pronuclear injection, and founders (C57BL/6) were identified by genotyping and qPCR for transgene expression levels. Based on genotyping, the TG-founders (hNrf2, both male/female) were confirmed, and breeding colonies were maintained separately under controlled conditions in the Animal Research Facility at the University of Utah and University of Alabama at Birmingham. caNrf2-TGH mice at the age of $\sim 1.5$ months were treated with buthionine sulfoximine, an inhibitor of gamma-glutamylcysteine synthetase (GCLC), which is involved in GSH synthesis.

\section{Necropsy and tissue collection}

Mice (2-18 months of age, $n=4-6 /$ group/gender) were anesthetized with isoflurane and euthanized by cervical dis- location. Hearts were immediately excised, perfused with ice-cold phosphate-buffered saline (PBS), and cut along the coronal plane. Two to three cross sections were obtained and stored in $10 \%$ zinc formalin and/or optimal cutting temperature (OCT) medium for immunohistochemistry/immunofluorescence imaging. The apex tissue biopsy ( $\sim 20 \mathrm{mg} /$ each) was collected for RNA studies and stored in $300 \mu \mathrm{L}$ of RNAlater solution. Before paraffin embedding and sectioning, tissue samples were kept in $10 \%$ zinc formalin for $24 \mathrm{~h}$.

\section{RNA isolation, next-generation RNA sequencing, and real-time $q P C R$}

Heart tissue from NTG, caNrf2 TGL, and TGH $(n=6)$ at 6 months of age was homogenized using the QIAshredder kit, and RNA was subsequently extracted with RNeasy mini kits according to the manufacturer's instructions. Concentrations of freshly isolated RNA were obtained using a NanoDrop $\mathrm{ONE}^{\mathrm{c}}$ (Thermo Scientific, Waltham, MA). Two micrograms of RNA from each sample ( $n=3-4$ /group) was used to perform next-generation RNA sequencing and analyzed as previously described (42), and $1.25 \mu \mathrm{g}$ of RNA was used to synthesize cDNA with a QuantiTect reverse transcription kit. Twenty-five to $50 \mathrm{ng}$ of cDNA template was used with $1 \mathrm{pmol}$ primer in a $10 \mu \mathrm{L}$ SYBR green reaction mix (204056; Qiagen) and was amplified in a Roche LightCycler 480 (Roche, Basel, Switzerland). Primers were designed with NCBI primer tools (Table 1 for sequences) and procured from IDT. Real-time qPCR was performed for randomly selected genes represented in heatmaps to valid the RNA sequencing, relative expression was quantified using $\mathrm{Ct}$ values, and expression fold-change was calculated by normalization to the $\mathrm{Ct}$ of housekeeping genes Gapdh or Arbpl using the $2^{-\Delta \Delta \mathrm{Ct}}$ method $(45,47,53)$.

\section{Protein isolation and IB}

Heart tissues from NTG, caNrf2 TGL, and TGH mice $(n=4-8)$ at 3-18 months of age were homogenized in a cytosolic extraction buffer $(10 \mathrm{~m} M$ HEPES, $10 \mathrm{~m} M \mathrm{KCl}$, $0.1 \mathrm{~m} M$ EDTA, $0.5 \mathrm{~m} M \mathrm{MgCl}_{2}$, with freshly prepared $0.1 \mathrm{~m} M$ phenyl methylsulfonyl fluoride (PMSF), $1 \mathrm{~m} M$ dithiothreitol, and $1 \%$ Triton $\mathrm{X}-100, \mathrm{pH} 7.9)$ and centrifuged at $5000 \mathrm{rpm}$ for 5-6 min. The pellet was processed to obtain nuclear fractions using nuclear extraction buffer $(20 \mathrm{mM}$ HEPES, $420 \mathrm{~m} M \mathrm{NaCl}, 0.1 \mathrm{~m} M$ EDTA, $1.5 \mathrm{~m} M \mathrm{MgCl}_{2}, 25 \%$ glycerol with $0.5 \mathrm{~m} M$ PMSF, and $1 \mathrm{~m} M$ dithiothreitol, $\mathrm{pH}$ 7.9) and centrifuged at $8200 \mathrm{rpm}$ for $10 \mathrm{~min}$ as previously described $(26,45,47,53)$. Protein concentrations were

Table 1. Complete List of Real-Time Quantitative Polymerase Chain Reaction Primer Sequences

\begin{tabular}{|c|c|c|}
\hline Gene name & Forward $\left(5^{\prime} \ldots 3^{\prime}\right)$ & Reverse $\left(5^{\prime} \ldots . .3^{\prime}\right)$ \\
\hline Gclm & CTTCGCCTCCGATTGAAGATG & AAAGGCAGTCAAATCTGGTGG \\
\hline Nqo1 & AGGATGGGAGGTACTCGAATC & TGCTAGAGATGACTCGGAAGG \\
\hline Gsr & CACGGCTATGCAACATTCGC & GTGTGGAGCGGTAAACTTTTTC \\
\hline Gst- $\alpha 4$ & TGATTGCCGTGGCTCCATTTA & CAACGAGAAAAGCCTCTCCGT \\
\hline Nppa & GCTTCCAGGCCATATTGGAG & GGGGGCATGACCTCATCTT \\
\hline$N p p b$ & GAGGTCACTCCTATCCTCTGG & GCCATTTCCTCCGACTTTTCTC \\
\hline Myh6 & GAGTGGGAGTTTATCGACTTCG & CCTTGACATTGCGAGGCTTC \\
\hline Myh7 & ACTGTCAACACTAAGAGGGTCA & TTGGATGATTTGATCTTCCAGGG \\
\hline Gapdh & TGACCTCAACTACATGGTCTACA & CTTCCCATTCTCGGCCTTG \\
\hline Arbpl & TGAGATTCGGGATATGCTGTTGG & CGGGTCCTAGACCAGTGTTCT \\
\hline
\end{tabular}


Table 2. Complete List of Primary ANTIBODIES AND SOURCES

\begin{tabular}{|c|c|c|}
\hline Antibodies & Source & Identifier \\
\hline Anti-mouse NRF2 & $\begin{array}{l}\text { Santa Cruz } \\
\text { biotechnology }\end{array}$ & Cat. No. sc-722 \\
\hline bbit GSR & Abcam & Cat. No. AB16801 \\
\hline Anti-rabbit GST- $\mu$ & Abcam & Cat. No. AB178684 \\
\hline Anti-rabbit NQO1 & Abcam & Cat. No. AB34173 \\
\hline bbit GCLC & Abcam & Cat. No. AB41463 \\
\hline Anti-rabbit CAT & EMD Millip & Cat. No. 219010 \\
\hline Anti-rabbit GPX1 & Abcam & Cat. No. AB22604 \\
\hline it SOD1 & $\mathrm{Abc}$ & Cat. No. AB13498 \\
\hline t GCLM & Abcam & Cat. No. AB8 \\
\hline it SOD2 & Abcam & Cat. No. AB1 \\
\hline Ant & $\begin{array}{l}\text { Dr. Ronald } \\
\text { Mason }\end{array}$ & N/A \\
\hline $\begin{array}{l}\text { Anti-mouse } \\
\text { GSH-NEM }\end{array}$ & EMD Millipore & Cat. No. 1 \\
\hline
\end{tabular}

determined with the Bradford reagent, and equal amounts of protein were resolved on $10 \%-12 \%$ sodium dodecyl sulfatepolyacrylamide gel electrophoresis. Proteins were then transferred to polyvinylidene difluoride membranes (EMD Millipore Corp., Billerica, MA) and blocked in tris-buffered saline-Tween 20 (TBST) containing 5\%-10\% nonfat dry milk or BSA. Membranes were then incubated overnight at $4^{\circ} \mathrm{C}$ or $2 \mathrm{~h}$ at room temperature with their respective primary antibodies, including antibodies against GCLM, GCLC, NQO1, GSR, GST $\mu$, SOD1, SOD2, GPX1, NRF2, and CAT proteins (see Table 2 for details). The primary antibodies were diluted with $1 \%-2 \%$ BSA in TBST for incubation. Following $2 \times 10$-min washes with TBST, horseradish peroxidase IgG (Vector Laboratories)-conjugated secondary antibody (anti-rabbit and anti-mouse) incubation followed by electrochemiluminescence-based detection (Pierce, Rockford, IL) of antigen/antibody complex was performed, and the signals were captured using autoradiography films or images were obtained with an Amersham Imager 600 (GE Healthcare Life Sciences, Chicago, IL). Bio-Rad dual-color protein standards (\#1610374) were compared to determine the size of the proteins. Protein loading was monitored by stripping the membrane with a mild stripping buffer $(1.5 \%$ glycine, $0.1 \%$ SDS, and $1 \%$ Tween 20 ) and reprobing for glyceraldehyde 3-phosphate dehydrogenase (GAPDH)/ LAMIN-B1 expression. The signal densitometry was quantified using ImageJ software and then normalized to the intensity of NTG sample (housekeeping protein GAPDH as internal standard). Mean arbitrary units normalized with GAPDH were used to calculate protein fold-change in TGL and TGH mice relative to NTG ( $n=4-6$ /group).

\section{Nrf2 binding activity assay}

Efficiency of Nrf2 DNA-binding activity in NTG and TG mice hearts ( $n=6$ /group) was evaluated at the age of 6-8 months using a commercially available Trans AM Nrf2 kit (Active Motif) as previously described (53). Briefly, the nuclear protein extracts $(10 \mu \mathrm{g})$ were incubated with immobilized wild-type or mutated competitor oligonucleotides bearing the ARE consensus sequence. The bound Nrf 2 was detected using an anti-Nrf2 primary antibody $(100 \mu \mathrm{L}$ of a
1:1000 dilution) reaction followed by washing and incubation with the horseradish peroxidase-conjugated secondary antibody (100 $\mu \mathrm{L}$ of a 1:1000 dilution) before chromogenic reaction with the tetramethylbenzidine substrate. The color forming reaction was terminated by addition of stop solution and the optical density was read at $450 \mathrm{~nm}$. Incubation with normal rabbit polyclonal IgG was also performed separately to determine the specificity of the Nrf2 antibody.

\section{Characterization of redox status markers}

GSH redox measurements. Reduced GSH and oxidized GSH (GSSG) levels of NTG and caNrf2-TG hearts were measured using a GSH assay kit (Cat. No. 703002; Cayman Chemical, Ann Arbor, MI). Myocardial 2-(N-morpholino) ethanesulfonic acid (MES) lysates were prepared in MES buffer (acidic; pH 6.0) and centrifuged for $5 \mathrm{~min}$ at $5000 \mathrm{rpm}$ in $4^{\circ} \mathrm{C}$. An aliquot of the supernatant was used for protein determination and equal volume of $10 \%$ meta-phosphoric acid (MPA; Cat. No. 239275; Sigma) was added to the remaining samples to precipitate the proteins. The supernatants were equally mixed with $10 \%$ MPA to precipitate proteins. The MPA extracts were then treated with $4 M$ triethanolamine (TEAM) reagent. The TEAM-treated extracts were aliquoted for GSH and GSSG quantification. The extracts for GSSG measurements were further processed with $1 M$ 2vinylpyridine, and the enzymatic recycling assay was executed according to the manufacturer's instructions. The GSH and GSSG standards were prepared and treated in the same way with the heart samples and the redox state was determined $(26,38,43,47,53)$.

\section{High-performance liquid chromatography-based assay of thiol/disulfide redox in the cardiac tissue}

Upon tissue collection, $\sim 50 \mathrm{mg}$ of cardiac tissue from NTG and caNrf2-TG mice ( $n=6 /$ group) was added to $0.5 \mathrm{~mL}$ of $5 \%(\mathrm{w} / \mathrm{v})$ perchloric acid containing $0.2 \mathrm{M}$ boric acid and $10 \mu M \gamma$-Glu-Glu (internal standard) solution. The sample was briefly sonicated on ice and centrifuged for $5 \mathrm{~min}$ at $5000 \mathrm{rpm}$ in $4^{\circ} \mathrm{C}$. The supernatant was aliquoted $(300 \mu \mathrm{L} /$ tube) and stored at $-80^{\circ} \mathrm{C}$ until derivatization, which was conducted at the Emory Clinical Biomarkers laboratory. The protein pellet was further resuspended in $200 \mu \mathrm{L} 1 \mathrm{~N} \mathrm{NaOH}$ and subjected to protein assay (23-25).

Derivatization. A $60 \mu \mathrm{L}$ aliquot of $50 \mathrm{~m} M$ iodoacetic acid solution was then added to the $300 \mu \mathrm{L}$ supernatant aliquot. The samples were vortexed, and $\mathrm{pH}$ adjusted to $9.0 \pm 0.2$ with $1 \mathrm{~N} \mathrm{KOH} /$ tetraborate solution. The tubes were incubated at room temperature for $20 \mathrm{~min}$ for complete precipitation of potassium perchlorate. Following incubation, $300 \mu \mathrm{L}$ of $75 \mathrm{~m} M$ dansyl chloride solution (prepared in acetone) was added to the tubes, and samples were vortexed well and stored in the dark at room temperature for $20 \mathrm{~h}$. Following derivatization, chloroform $(500 \mu \mathrm{L})$ was added to each tube, vortexed, centrifuged, and samples stored at $4^{\circ} \mathrm{C}$ in the dark until HPLC analysis.

HPLC analysis. Samples were centrifuged for 2 min and an aliquot of the upper aqueous layer was transferred to an autosampler vial. The instrumentation used is a gradient HPLC module 2695 (Waters) with Empower software. The Waters 
2475 multifluorescence detector was used with excitation at $335 \mathrm{~nm}$ and emission detected at $518 \mathrm{~nm}$. Separations were obtained on a $25 \mathrm{~cm}$ by $4.6 \mathrm{~mm}, 5 \mu \mathrm{m}$ silica, LC-NH2 Supelco column from Sigma Aldrich (Cat No. 58338). Flow rate for HPLC was maintained constant at $1.0 \mathrm{~mL} / \mathrm{min}$. The solvent gradient used was as follows: initial solvent conditions were $80 \%$ A $[80 \%(\mathrm{v} / \mathrm{v})$ methanol/water] and $20 \%$ B [acetatebuffered ( $\mathrm{pH} 4.6)$ methanol solution] run at $1 \mathrm{~mL} / \mathrm{min}$ for 10 min. A linear gradient to $20 \% \mathrm{~A}$ and $80 \% \mathrm{~B}$ was run over the period from 10 to $30 \mathrm{~min}$. From 30 to $46 \mathrm{~min}$, the conditions were maintained at $20 \% \mathrm{~A}$ and $80 \% \mathrm{~B}$ and returned to $80 \% \mathrm{~A}$ and $20 \% \mathrm{~B}$ from 46 to $48 \mathrm{~min}$. Equilibration time for the next run was $12 \mathrm{~min}$ [Solvent A: $80 \%(\mathrm{v} / \mathrm{v})$ methanol/water and Solvent B: acetate-buffered ( $\mathrm{pH} 4.6)$ methanol solution prepared by mixing $640 \mathrm{~mL}$ of methanol, $200 \mathrm{~mL}$ of acetate stock, $125 \mathrm{~mL}$ of glacial acetic acid, and $50 \mathrm{~mL}$ of water].

Calculations. Integrated peaks provided by the HPLC software corresponding to GSH, GSSG, Cys, CySS, and mixed disulfide (CySSG) were used and concentrations were calculated relative to the integrated peak of the internal standard $(\gamma$-Glu-Glu) and normalized to the protein concentration. The individual concentrations, expressed in molar values, were used with the Nernst equation to calculate redox potentials. At $\mathrm{pH} 7.4$, an Eo value of $-264 \mathrm{mV}$ was used for the GSH/GSSG couple, and an Eo value of $-250 \mathrm{mV}$ was used for the Cys/CySS couple.

\section{Assessment of total antioxidant activity}

Myocardial TAC was determined in homogenates of NTG, caNrf2 TGL, and TGH mice using the TAC assay kit (OxiSelect: STA-360; Cell Biolabs, Inc.) according to the manufacturer's instructions. The conversion of copper (II) ion $(\mathrm{Cu} 2+)$ into copper $(\mathrm{I})$ ion $(\mathrm{Cu}+)$ by the antioxidants was measured as total reducing capacity of the nonenzymatic antioxidants present in the myocardial extracts. In brief, heart extracts were prepared immediately upon tissue harvesting ( $n=6-8$ /group). Equal amounts of heart tissues $(20-25 \mathrm{mg}$ ) were homogenized with $200 \mu \mathrm{L}$ of ice-cold PBS, centrifuged, and $20 \mu \mathrm{L}$ of supernatant was mixed with $180 \mu \mathrm{L}$ of reaction buffer; initial absorbance was measured at $490 \mathrm{~nm}$. Copper ion reagent $(50 \mu \mathrm{L})$ was then added to the mixture to initiate the reaction and incubated on an orbital shaker for $5 \mathrm{~min}$ and $50 \mu \mathrm{L}$ of $1 \times$ stop solution was added to stop the reaction. The color development was measured at $490 \mathrm{~nm}$. Antioxidant capacity was represented as fold change in relation to NTG (53).

\section{Immunofluorescent microscopy}

General protocol. For immunofluorescent staining $(n=$ 3-6/group), heart tissue was embedded in O.C.T. freezing medium and $10 \mu \mathrm{m}$ cryostat sections were obtained. In preparation for staining, sections were incubated with $4 \%$ paraformaldehyde for $15 \mathrm{~min}$ and washed thrice with PBS followed by permeabilization with $0.25 \%$ Triton X-100. Following three washes with PBS, tissue sections were blocked with 5\% normal goat serum in PBS for $1 \mathrm{~h}$ to prevent nonspecific binding of antibodies. Sections were then incubated for $1 \mathrm{~h}$ at room temperature or overnight at $4^{\circ} \mathrm{C}$ with primary antibodies. Following primary antibody incubation, all sections were washed with PBS thrice and incubated with one of the following secondary antibodies at 1:1000 for $1 \mathrm{~h}$ at room temperature: Alexa Fluor 488 goat anti-mouse $\operatorname{IgM}$ ( $\mu$-chain) (A21042), anti-rabbit IgG (H+L) (A11008), or Alexa Fluor 647 chicken anti-mouse IgG2a (y2a) (A21241), anti-rabbit IgG (H+L) (A21443) (Life Technologies, Carlsbad, CA). Finally, the sections were washed thoroughly with PBS $(3 \times 10 \mathrm{~min}$ each $)$ and mounted with Fluoroshield/DAPI (ab104139; Abcam, Cambridge, MA). Images were acquired with an Olympus BX43 fluorescent microscope or with a Nikon A1 confocal microscope using a $60 \times$ objective. At least three to four micrographs were taken for each section and used to calculate the appropriate intensity for each target $(18,52-54)$.

WGA staining. Cryostat sections used for WGA staining were labeled with $5 \mu \mathrm{g} / \mathrm{mL}$ WGA in PBS (Alexa Fluor 594 Conjugate, W11262; Molecular Probes, Eugene, OR) in a light-protected chamber maintained at $37^{\circ} \mathrm{C}$ for $30 \mathrm{~min}$. After washing and fixation with mounting medium, cardiac cells were imaged with a confocal microscope set to $60 \times$. Cardiomyocyte cell size was measured using NIH ImageJ software (52).

DMPO staining. For the evaluation of myocardial DMPO protein adducts, mice were injected intraperitoneally with two $1 \mathrm{~g} / \mathrm{kg}$ doses of DMPO (D048-10; Dojindo Molecular Technologies, Inc., Kumamoto, Japan) at 2 and $1 \mathrm{~h}$ before sacrificing. DMPO is a free-radical spin trap used to detect the free radical in the biological samples. DMPO staining in tissues/ cells using anti-DMPO antibodies detects DMPO-protein adducts, an indirect estimation of oxidant levels (ROS) (52). The procured hearts were fixed in $10 \%$ neutral buffered formalin and soaked in $30 \%$ sucrose for $24 \mathrm{~h}$. Then, the tissues were fixed in cryostat OCT solutions and stored at $-20^{\circ} \mathrm{C}$ before sectioning. Cryostat sections $(5 \mu M)$ were prepared and blocked with $1 \%$ casein PBS for $1 \mathrm{~h}$ at room temperature. Slides were then incubated overnight at $4^{\circ} \mathrm{C}$ with $5 \mu \mathrm{g} / \mathrm{mL}$ mouse anti-DMPO primary antibody (kindly provided by Dr. Mason). Sections were washed, conjugated with secondary antibodies, and mounted with DAPI. Images were obtained with a confocal microscope at $60 \times$ magnification.

DHE staining for the determination of tissue level of ROS superoxide. The DHE, a lipophilic, cell-permeable, red fluorogenic dye, was used to measure the level of ROS (superoxide only). Briefly, the frozen sections $(10 \mu \mathrm{m})$ of the myocardium were incubated with $5 \mu \mathrm{g} / \mathrm{mL}$ of DHE in PBS in a light-protected chamber maintained at $37^{\circ} \mathrm{C}$ for $30 \mathrm{~min}$. Then tissue sections were washed with PBS for two to three times to remove the excess DHE stain and then fixed with the Fluoroshield mounting medium. The images were captured by randomly selecting 3-5 fields/section using an Olympus BX43 fluorescent microscope $(18,53,54)$.

\section{Noninvasive echocardiography analysis of cardiac function}

NTG (at 1.5, 3, 6, and $>15$ months of age), caNrf2-TG (at $\sim 6$ and $>15$ months of age), and DTG (hNrf2xmcaNrf 2 at $\sim 2$ months of age) mice were anesthetized with $1 \%-2 \%$ isoflurane, supplemented with $100 \%$ oxygen, and chest area was shaved in preparation for echocardiography analyses $(n=5-10)$ using the Vevo2100 Imaging System (Fujifilm VisualSonics, Inc., Ontario, Canada). A $38 \mathrm{MHz}$ probe was 
used to capture images at maximum $(50 \mu M)$ resolution. Long-axis B-mode was used for strain analysis to calculate $\mathrm{EF}$, end diastole/systole left ventricular mass. The parasternal short-axis M-mode was utilized in the determination of fractional shortening, wall thickness, and chamber dimension during systole and diastole. Pulse Wave/Color Doppler analysis was used to measure early (E) filling and late atrial (A) filling velocity, as well as $\mathrm{MV}$ tissue motion ( $\left.\mathrm{E}^{\prime}\right)$ in systole and diastole. Three consecutive cardiac cycles from $\mathrm{B}$ - and M-mode images were used for measuring for each variable $(43,52)$.

\section{Statistical analysis}

All data are represented as mean \pm standard error of the mean. When three or more groups were analyzed, a one-way ANOVA with post hoc Tukey multiple comparison test was performed. Student's $t$-test was used to calculate statistical significance. All analyses were performed using GraphPad Prism 7. Significance: $* p<0.05$; $* * p<0.01$; $* * * p<0.001$; and ns, no significance.

\section{Acknowledgments}

The authors thank Dr. James Fang, MD, and Dr. John Michael, MD, for providing resources at the University of Utah. They acknowledge Dr. Ivor Benjamin and Dr. Jeffrey Robbins for providing the mutant-CryAB mice, CryABantibodies, $\alpha$-MHC promoter constructs. The authors also thank Dr. Sethu Palaniappan for providing the fluorescence microscope facility. They are grateful to Dr. Susan Tamowski, Director, Transgene-Targeting Mouse Facility, for her excellent technical support to generate the caNrf2-TG mice. They also thank Dr. Michael Crowley (Heflin Center for Genomic Sciences at UAB) for providing access to the genomic core facilities. The authors acknowledge the support from Mr. Wayne E. Bradley for assisting with analyses/interpretation of echocardiography. They thank Dr. Madhusudhanan Narasimhan for editorial assistance.

\section{Author Disclosure Statement}

No competing financial interests exist.

\section{Funding Information}

This study was supported by funding from NHLBI (2HL118067 and HL118067), NIA (AG042860), the AHA (BGIA 0865015F), the University of Utah Center for Aging Pilot grant (2009), the Division of Cardiovascular Medicine/ Department of Medicine, University of Utah, and the start-up funds (for N.S.R.) by the Department of Pathology and School of Medicine, the University of Alabama at Birmingham, AL, and UABAMC21 grant by the University of Alabama at Birmingham, AL.

\section{Supplementary Material}

Supplementary Figure S1 Supplementary Figure S2 Supplementary Figure S3 Supplementary Figure S4 Supplementary Figure S5
Supplementary Figure S6

Supplementary Figure S7

Supplementary Figure S8

Supplementary Figure S9

Supplementary Video S1

Supplementary Video S2

Supplementary Video S3

\section{References}

1. Ago T, Liu T, Zhai P, Chen W, Li H, Molkentin JD, Vatner SF, and Sadoshima J. A redox-dependent pathway for regulating class II HDACs and cardiac hypertrophy. Cell 133: 978-993, 2008.

2. Al-Khudairy L, Flowers N, Wheelhouse R, Ghannam O, Hartley L, Stranges S, and Rees K. Vitamin C supplementation for the primary prevention of cardiovascular disease. Cochrane Database Syst Rev 3: CD011114, 2017.

3. Bairati I, Meyer F, Jobin E, Gelinas M, Fortin A, Nabid A, Brochet F, and Tetu B. Antioxidant vitamins supplementation and mortality: a randomized trial in head and neck cancer patients. Int J Cancer 119: 2221-2224, 2006.

4. Bansal D, Bhalla A, Bhasin DK, Pandhi P, Sharma N, Rana S, and Malhotra S. Safety and efficacy of vitamin-based antioxidant therapy in patients with severe acute pancreatitis: a randomized controlled trial. Saudi J Gastroenterol 17: 174-179, 2011.

5. Bhuiyan $\mathrm{T}$ and Maurer MS. Heart failure with preserved ejection fraction: persistent diagnosis, therapeutic enigma. Curr Cardiovasc Risk Rep 5: 440-449, 2011.

6. Bjelakovic G, Nikolova D, Gluud LL, Simonetti RG, and Gluud C. Mortality in randomized trials of antioxidant supplements for primary and secondary prevention: systematic review and meta-analysis. JAMA 297: 842-857, 2007.

7. Borlaug BA and Paulus WJ. Heart failure with preserved ejection fraction: pathophysiology, diagnosis, and treatment. Eur Heart J 32: 670-679, 2011.

8. Brewer AC, Mustafi SB, Murray TV, Rajasekaran NS, and Benjamin IJ. Reductive stress linked to small HSPs, G6PD, and Nrf2 pathways in heart disease. Antioxid Redox Signal 18: 1114-1127, 2013.

9. Briancon S, Boini S, Bertrais S, Guillemin F, Galan P, and Hercberg S. Long-term antioxidant supplementation has no effect on health-related quality of life: the randomized, double-blind, placebo-controlled, primary prevention SU.VI.MAX trial. Int J Epidemiol 40: 1605-1616, 2011.

10. Bui AL, Horwich TB, and Fonarow GC. Epidemiology and risk profile of heart failure. Nat Rev Cardiol 8: 30-41, 2011.

11. Burkhoff D, Maurer MS, and Packer M. Heart failure with a normal ejection fraction: is it really a disorder of diastolic function? Circulation 107: 656-658, 2003.

12. Chan JY and Chan SH. Activation of endogenous antioxidants as a common therapeutic strategy against cancer, neurodegeneration and cardiovascular diseases: a lesson learnt from DJ-1. Pharmacol Ther 156: 69-74, 2015.

13. Cho HY, Marzec J, and Kleeberger SR. Functional polymorphisms in Nrf2: implications for human disease. Free Radic Biol Med 88: 362-372, 2015.

14. Cohn JN, Ferrari R, and Sharpe N. Cardiac remodelingconcepts and clinical implications: a consensus paper from an international forum on cardiac remodeling. Behalf of an 
International Forum on Cardiac Remodeling. J Am Coll Cardiol 35: 569-582, 2000.

15. Cook NR, Albert CM, Gaziano JM, Zaharris E, MacFadyen J, Danielson E, Buring JE, and Manson JE. A randomized factorial trial of vitamins $\mathrm{C}$ and $\mathrm{E}$ and beta carotene in the secondary prevention of cardiovascular events in women: results from the Women's Antioxidant Cardiovascular Study. Arch Intern Med 167: 1610-1618, 2007.

16. Dhalla NS, Temsah RM, and Netticadan T. Role of oxidative stress in cardiovascular diseases. J Hypertens 18 : 655-673, 2000.

17. Eggler AL, Small E, Hannink M, and Mesecar AD. Cul3mediated Nrf2 ubiquitination and antioxidant response element (ARE) activation are dependent on the partial molar volume at position 151 of Keap1. Biochem J 422: 171-180, 2009.

18. Gounder SS, Kannan S, Devadoss D, Miller CJ, Whitehead KJ, Odelberg SJ, Firpo MA, Paine R, III, Hoidal JR, Abel $\mathrm{ED}$, and Rajasekaran NS. Impaired transcriptional activity of Nrf2 in age-related myocardial oxidative stress is reversible by moderate exercise training. PLoS One 7: e45697, 2012.

19. Griffith OW and Meister A. Potent and specific inhibition of glutathione synthesis by buthionine sulfoximine (S-nbutyl homocysteine sulfoximine). 254: 7558-7560, 1979.

20. Hariharan N, Zhai P, and Sadoshima J. Oxidative stress stimulates autophagic flux during ischemia/reperfusion. Antioxid Redox Signal 14: 2179-2190, 2011.

21. Huppke P, Weissbach S, Church JA, Schnur R, Krusen M, Dreha-Kulaczewski S, Kuhn-Velten WN, Wolf A, Huppke B, Millan F, Begtrup A, Almusafri F, Thiele H, Altmuller J, Nurnberg P, Muller M, and Gartner J. Activating de novo mutations in NFE2L2 encoding NRF2 cause a multisystem disorder. Nat Commun 8: 818, 2017.

22. Jain $\mathrm{A}$, Mårtensson $\mathrm{J}$, Stole $\mathrm{E}$, Auld $\mathrm{PA}$, and Meister A. Glutathione deficiency leads to mitochondrial damage in brain. Proc Natl Acad Sci U S A 88: 1913-1917, 1991.

23. Jones DP. Redox potential of GSH/GSSG couple: assay and biological significance. Methods Enzymol 348: 93-112, 2002.

24. Jones DP, Carlson JL, Samiec PS, Sternberg P, Jr., Mody VC, Jr., Reed RL, and Brown LA. Glutathione measurement in human plasma. Evaluation of sample collection, storage and derivatization conditions for analysis of dansyl derivatives by HPLC. Clin Chim Acta 275: 175-184, 1998.

25. Jones DP and Liang Y. Measuring the poise of thiol/disulfide couples in vivo. Free Radic Biol Med 47: 13291338, 2009.

26. Kannan S, Muthusamy VR, Whitehead KJ, Wang L, Gomes AV, Litwin SE, Kensler TW, Abel ED, Hoidal JR, and Rajasekaran NS. Nrf2 deficiency prevents reductive stress-induced hypertrophic cardiomyopathy. Cardiovasc Res 100: 63-73, 2013.

27. Kensler TW, Egner PA, Agyeman AS, Visvanathan K, Groopman JD, Chen J-G, Chen T-Y, Fahey JW, and Talalay P. Keap1-Nrf2 signaling: a target for cancer prevention by sulforaphane. Top Curr Chem 329: 163-177, 2013.

28. Klapholz M, Maurer M, Lowe AM, Messineo F, Meisner JS, Mitchell J, Kalman J, Phillips RA, Steingart R, Brown Jr EJ, Berkowitz R, Moskowitz R, Soni A, Mancini D, Bijou R, Sehhat K, Varshneya N, Kukin M, Katz SD, Sleeper LA, and Le Jemtel TH. Hospitalization for heart failure in the presence of a normal left ventricular ejection fraction: results of the New York heart failure registry. $J$ Am Coll Cardiol 43: 1432-1438, 2004.
29. Kobayashi A, Kang MI, Watai Y, Tong KI, Shibata T, Uchida K, and Yamamoto M. Oxidative and electrophilic stresses activate Nrf2 through inhibition of ubiquitination activity of Keap1. Mol Cell Biol 26: 221-229, 2006.

30. Kris-Etherton PM, Lichtenstein AH, Howard BV, Steinberg D, and Witztum JL. Antioxidant vitamin supplements and cardiovascular disease. Circulation 110: 637-641, 2004.

31. Kwak MK and Kensler TW. Targeting NRF2 signaling for cancer chemoprevention. Toxicol Appl Pharmacol 244: 6676, 2010

32. Lee JM, Li J, Johnson DA, Stein TD, Kraft AD, Calkins MJ, Jakel RJ, and Johnson JA. Nrf2, a multi-organ protector? FASEB J 19: 1061-1066, 2005.

33. Martensson J, Jain A, Stole E, Frayer W, Auld PA, and Meister A. Inhibition of glutathione synthesis in the newborn rat: a model for endogenously produced oxidative stress. Proc Natl Acad Sci U S A 88: 9360-9364, 1991.

34. McGee AM and Baines CP. Complement 1q-binding protein inhibits the mitochondrial permeability transition pore and protects against oxidative stress-induced death. Biochem J 433: 119-125, 2011.

35. Meister A. Glutathione deficiency produced by inhibition of its synthesis, and its reversal; applications in research and therapy. Pharmacol Ther 51: 155-194, 1991.

36. Miljkovik LV and Spiroska V. Heart failure with preserved ejection fraction-concept, pathophysiology, diagnosis and challenges for treatment. Open Access Maced J Med Sci 3: 521-527, 2015.

37. Morita H, Nagai R, Seidman JG, and Seidman CE. The impact of genetic testing on hypertrophic cardiomyopathy and heart failure (As of 12072009 by HIRO). J Cardiovasc Transl Res 3: 297-303, 2010.

38. Muthusamy VR, Kannan S, Sadhaasivam K, Gounder SS, Davidson CJ, Boeheme C, Hoidal JR, Wang L, and Rajasekaran NS. Acute exercise stress activates Nrf2/ARE signaling and promotes antioxidant mechanisms in the myocardium. Free Radic Biol Med 52: 366-376, 2012.

39. Nagueh SF, Appleton CP, Gillebert TC, Marino PN, Oh JK, Smiseth OA, Waggoner AD, Flachskampf FA, Pellikka PA, and Evangelista A. Recommendations for the evaluation of left ventricular diastolic function by echocardiography. J Am Soc Echocardiogr 22: 107-133, 2009.

40. Narasimhan M and Rajasekaran NS. Reductive potentiala savior turns stressor in protein aggregation cardiomyopathy. Biochim Biophys Acta 1852: 53-60, 2015.

41. Palliyaguru DL, Chartoumpekis DV, Wakabayashi N, Skoko JJ, Yagishita Y, Singh SV, and Kensler TW. Withaferin A induces Nrf2-dependent protection against liver injury: role of Keap1-independent mechanisms. Free Radic Biol Med 101: 116-128, 2016.

42. Quiles JM, Narasimhan M, Mosbruger T, Shanmugam G, Crossman D, and Rajasekaran NS. Identification of transcriptome signature for myocardial reductive stress. Redox Biol 13: 568-580, 2017.

43. Rajasekaran NS, Connell P, Christians ES, Yan LJ, Taylor RP, Orosz A, Zhang XQ, Stevenson TJ, Peshock RM, Leopold JA, Barry WH, Loscalzo J, Odelberg SJ, and Benjamin IJ. Human alpha B-crystallin mutation causes oxido-reductive stress and protein aggregation cardiomyopathy in mice. Cell 130: 427-439, 2007.

44. Rajasekaran NS, Devaraj NS, and Devaraj H. Modulation of rat erythrocyte antioxidant defense system by buthionine sulfoximine and its reversal by glutathione monoester therapy. Biochim Biophys Acta 1688: 121-129, 2004. 
45. Rajasekaran NS, Firpo MA, Milash BA, Weiss RB, and Benjamin IJ. Global expression profiling identifies a novel biosignature for protein aggregation R120GCryAB cardiomyopathy in mice. Physiol Genomics 35: 165-172, 2008.

46. Rajasekaran NS, Sathyanarayanan S, Devaraj NS, and Devaraj H. Chronic depletion of glutathione (GSH) and minimal modification of LDL in vivo: its prevention by glutathione mono ester (GME) therapy. Biochim Biophys Acta 1741: 103-112, 2005.

47. Rajasekaran NS, Varadharaj S, Khanderao GD, Davidson CJ, Kannan S, Firpo MA, Zweier JL, and Benjamin IJ. Sustained activation of nuclear erythroid 2-related factor 2/ antioxidant response element signaling promotes reductive stress in the human mutant protein aggregation cardiomyopathy in mice. Antioxid Redox Signal 14: 957-971, 2011.

48. Roma-Rodrigues $\mathrm{C}$ and Fernandes AR. Genetics of hypertrophic cardiomyopathy: advances and pitfalls in molecular diagnosis and therapy. Appl Clin Genet 7: 195-208, 2014.

49. Santos CX, Raza S, and Shah AM. Redox signaling in the cardiomyocyte: from physiology to failure. Int J Biochem Cell Biol 74: 145-151, 2016.

50. Schnabel R and Blankenberg S. Oxidative stress in cardiovascular disease: successful translation from bench to bedside? Circulation 116: 1338-1340, 2007.

51. Sen CK and Roy S. Relief from a heavy heart: redoxsensitive NF-kappaB as a therapeutic target in managing cardiac hypertrophy. Am J Physiol Heart Circ Physiol 289: H17-H19, 2005.

52. Shanmugam G, Narasimhan M, Conley RL, Sairam T, Kumar A, Mason RP, Sankaran R, Hoidal JR, and Rajasekaran NS. Chronic endurance exercise impairs cardiac structure and function in middle-aged mice with impaired Nrf2 signaling. Front Physiol 8, 2017.

53. Shanmugam G, Narasimhan M, Tamowski S, DarleyUsmar V, and Rajasekaran NS. Constitutive activation of Nrf2 induces a stable reductive state in the mouse myocardium. Redox Biol 12: 937-945, 2017.

54. Shelar SB, Narasimhan M, Shanmugam G, Litovsky SH, Gounder SS, Karan G, Arulvasu C, Kensler TW, Hoidal JR, Darley-Usmar VM, and Rajasekaran NS. Disruption of nuclear factor (erythroid-derived-2)-like 2 antioxidant signaling: a mechanism for impaired activation of stem cells and delayed regeneration of skeletal muscle. FASEB J30: 1865-1879, 2016.

55. Smyrnias I, Zhang X, Zhang M, Murray TV, Brandes RP, Schroder K, Brewer AC, and Shah AM. Nicotinamide adenine dinucleotide phosphate oxidase-4-dependent upregulation of nuclear factor erythroid-derived 2-like 2 protects the heart during chronic pressure overload. Hypertension 65: 547-553, 2015.

56. Sorriento D, De Luca N, Trimarco B, and Iaccarino G. The antioxidant therapy: new insights in the treatment of hypertension. Front Physiol 9: 258, 2018.

57. Steven S, Munzel T, and Daiber A. Exploiting the pleiotropic antioxidant effects of established drugs in cardiovascular disease. Int J Mol Sci 16: 18185-18223, 2015.

58. Taguchi K, Motohashi $\mathrm{H}$, and Yamamoto M. Molecular mechanisms of the Keap1-Nrf2 pathway in stress response and cancer evolution. Genes Cells 16: 123-140, 2011.

59. Tribble DL. AHA Science Advisory. Antioxidant consumption and risk of coronary heart disease: emphasison vitamin $\mathrm{C}$, vitamin $\mathrm{E}$, and beta-carotene: a statement for healthcare professionals from the American Heart Association. Circulation 99: 591-595, 1999.
60. Tsutsui $\mathrm{H}$, Kinugawa $\mathrm{S}$, and Matsushima S. Oxidative stress and heart failure. Am J Physiol Heart Circ Physiol 301: H2181-H2190, 2011.

61. Xin M, Olson EN, and Bassel-Duby R. Mending broken hearts: cardiac development as a basis for adult heart regeneration and repair. Nat Rev Mol Cell Biol 14: 529-541, 2013.

62. Yamamoto $M$, Kensler TW, and Motohashi $H$. The KEAP1-NRF2 System: a thiol-based sensor-effector apparatus for maintaining redox homeostasis. Physiol Rev 98: 1169-1203, 2018.

63. Youn JY, Siu KL, Lob HE, Itani H, Harrison DG, and Cai $\mathrm{H}$. Role of vascular oxidative stress in obesity and metabolic syndrome. Diabetes 63: 2344-2355, 2014.

64. Youn JY, Zhang J, Zhang Y, Chen H, Liu D, Ping P, Weiss $\mathrm{JN}$, and $\mathrm{Cai} \mathrm{H}$. Oxidative stress in atrial fibrillation: an emerging role of NADPH oxidase. J Mol Cell Cardiol 62: 72-79, 2013.

65. Zouein FA, de Castro Brás LE, da Costa DV, Lindsey ML, Kurdi M, and Booz GW. Heart failure with preserved ejection fraction: emerging drug strategies. J Cardiovasc Pharmacol 62: 13-21, 2013.

Address correspondence to: Dr. Namakkal S. Rajasekaran Division of Molecular and Cellular Pathology Department of Pathology Cardiac Aging and Redox Signaling Laboratory Center for Free Radical Biology The University of Alabama at Birmingham BMR2 Room 533, 901 19th Street South Birmingham, AL 35294-2180

USA

E-mail: rajnsr@uabmc.edu

Dr. Peipei Ping

Department of Physiology, Medicine/ Cardiology and Bioinformatics NIH BD2K Center of Excellence for Biomedical Computer at UCLA David Geffen School of Medicine at UCLA Los Angeles, CA 90095

USA

E-mail: pping38@g.ucla.edu

Date of first submission to ARS Central, May 16, 2019; date of final revised submission, February 12, 2020; date of acceptance, February 14, 2020.

$\begin{aligned} & \text { Abbreviations Used } \\ \alpha-\mathrm{MHC} & =\text { alpha-myosin heavy chain } \\ \mathrm{ARE} & =\text { antioxidant response element } \\ \mathrm{BSA} & =\text { bovine serum albumin } \\ \mathrm{BSO} & =\mathrm{L} \text {-buthionine-SR-sulfoximine } \\ \mathrm{caNrf} 2 & =\text { constitutively active Nrf } 2 \\ \mathrm{CAT} & =\text { catalase } \\ \mathrm{cRS} & =\text { chronic reductive stress } \\ \mathrm{DHE} & =\text { dihydroethidium }\end{aligned}$




\section{Abbreviations Used (Cont.)}

$\mathrm{DMPO}=5,5$-dimethyl-1-pyrroline $N$-oxide

$\mathrm{DTG}=$ double transgenic

$\mathrm{EF}=$ ejection fraction

GCLC $=$ glutamate-cysteine ligase catalytic

GPX1 = glutathione peroxidase 1

$\mathrm{GSH}=$ glutathione

$\mathrm{GSR}=$ glutathione reductase

GSSG $=$ oxidized glutathione

GST- $\mu=$ glutathione- $S$-transferase Mu (class)

$\mathrm{HCM}=$ hypertrophic cardiomyopathy

$\mathrm{HCM} i \mathrm{EF}=\mathrm{HCM}$ with abnormally increased ejection fraction

$\mathrm{HF}=$ heart failure

hNrf2 = human Nrf2

HPLC $=$ high-performance liquid chromatography

$\mathrm{IB}=$ immunoblotting

IDT $=$ Integrated DNA Technologies

IVSd $=$ end-diastolic interventricular septal thickness

$\begin{aligned} \mathrm{LVIDd} & =\text { end-diastolic } \mathrm{LV} \text { diameter } \\ \mathrm{LVVd} & =\text { end-diastolic LV volume } \\ \mathrm{MDA} & =\text { malondialdehyde } \\ \mathrm{MPA} & =\text { meta-phosphoric acid } \\ \mathrm{MV} & =\text { mitral valve } \\ \mathrm{NQO} 1 & =\text { NAD }(\mathrm{P}) \mathrm{H}: \text { Quinone Oxidoreductase } 1 \\ \text { Nrf2 (NFE2L2) } & =\text { nuclear factor (erythroid-derived-2)- } \\ \mathrm{NTG} & =\text { like } 2 \\ \mathrm{OCT} & =\text { optimal cutting temperature } \\ \mathrm{PBS} & =\text { phosphate-buffered saline } \\ \mathrm{PMSF} & =\text { phenyl methylsulfonyl fluoride } \\ \mathrm{PR} & =\text { proreductive } \\ \mathrm{ROS} & =\text { reactive oxygen species } \\ \mathrm{RS} & =\text { reductive stress } \\ \mathrm{SEM} & =\text { standard error of the mean } \\ \mathrm{TAC} & =\text { total antioxidant capacity } \\ \mathrm{TBST} & =\text { tris-buffered saline-Tween } 20 \\ \mathrm{TGH} & =\text { transgenic high } \\ \mathrm{TGL} & =\text { transgenic low } \\ \mathrm{WGA} & =\text { wheat germ agglutinin }\end{aligned}$

